AND THE MAIN FIELD OF STUDY

COMPUTER SCIENCE AND ENGINEERING,

SECOND CYCLE, 30 CREDITS

STOCKHOLM, SWEDEN 2020

\title{
Detecting Signal Corruptions in Voice Recordings for Speech Therapy
}

\author{
HELMER NYLÉN
}





\title{
Detecting Signal Corruptions in Voice Recordings for Speech Therapy
}

\author{
HELMER NYLÉN
}

Master's Programme, Computer Science, 120 credits Date: January 30, 2021

Supervisors: Sten Ternström and Saikat Chatterjee Examiner: Olov Engwall

School of Electrical Engineering and Computer Science Swedish title: Igenkänning av Signalproblem i Röstinspelningar för Logopedi 
Detecting Signal Corruptions in Voice Recordings for Speech Therapy / Igenkänning av Signalproblem i Röstinspelningar för Logopedi

(C) 2021 Helmer Nylén 


\section{Abstract}

When recording voice samples from a patient in speech therapy the quality of the recording may be affected by different signal corruptions, for example background noise or clipping. The equipment and expertise required to identify small disturbances are not always present at smaller clinics. Therefore, this study investigates possible machine learning algorithms to automatically detect selected corruptions in speech signals, including infrasound and random muting. Five algorithms are analyzed: kernel substitution based Support Vector Machine, Convolutional Neural Network, Long Short-term Memory (LSTM), Gaussian Mixture Model based Hidden Markov Model and Generative Model based Hidden Markov Model.

A tool to generate datasets of corrupted recordings is developed to test the algorithms in both single-label and multi-label settings. Mel-frequency Cepstral Coefficients are used as the main features. For each type of corruption different ways to increase the classification accuracy are tested, for example by using a Voice Activity Detector to filter out less relevant parts of the recording, changing the feature parameters, or using an ensemble of classifiers.

The experiments show that a machine learning approach is feasible for this problem as a balanced accuracy of at least $75 \%$ is reached on all tested corruptions. While the single-label study gave mixed results with no algorithm clearly outperforming the others, in the multi-label case the LSTM in general performs better than other algorithms. Notably it achieves over $95 \%$ balanced accuracy on both white noise and infrasound. As the algorithms are trained only on spoken English phrases the usability of this tool in its current state is limited, but the experiments are easily expanded upon with other types of audio recordings, corruptions, features, or classification algorithms.

\section{Keywords}

Noise, Classification algorithms, Audio recording, Machine learning, Acoustic signal processing 


\section{Sammanfattning}

När en patients röst spelas in för analys i talterapi kan inspelningskvaliteten påverkas av olika signalproblem, till exempel bakgrundsljud eller klippning. Utrustningen och expertisen som behövs för att upptäcka små störningar finns dock inte alltid tillgänglig på mindre kliniker. Därför undersöker denna studie olika maskininlärningsalgoritmer för att automatiskt kunna upptäcka utvalda problem i talinspelningar, bland andra infraljud och slumpmässig utsläckning av signalen. Fem algoritmer analyseras: stödvektormaskin, Convolutional Neural Network, Long Short-term Memory (LSTM), Gaussian mixture model-baserad dold Markovmodell och generatorbaserad dold Markovmodell.

Ett verktyg för att skapa datamängder med försämrade inspelningar utvecklas för att kunna testa algoritmerna. Vi undersöker separat fallen där inspelningarna tillåts ha en eller flera problem samtidigt, och använder framförallt en slags kepstralkoefficienter, MFCC.er, som särdrag. För varje typ av problem undersöker vi också sätt att förbättra noggrannheten, till exempel genom att filtrera bort irrelevanta delar av signalen med hjälp av en röstupptäckare, ändra särdragsparametrarna, eller genom att använda en ensemble av klassificerare.

Experimenten visar att maskininlärning är ett rimligt tillvägagångssätt för detta problem då den balanserade träffsäkerheten överskrider 75 \% för samtliga testade störningar. Den delen av studien som fokuserade på enproblemsinspelningar gav inga resultat som tydde på att en algoritm var klart bättre än de andra, men i flerproblemsfallet överträffade LSTM.en generellt övriga algoritmer. Värt att notera är att den nådde över $95 \%$ balanserad träffsäkerhet på både vitt brus och infraljud. Eftersom algoritmerna enbart tränats på engelskspråkiga, talade meningar så har detta verktyg i nuläget begränsad praktisk användbarhet. Däremot är det lätt att utöka dessa experiment med andra typer av inspelningar, signalproblem, särdrag eller algoritmer.

\section{Nyckelord}

Störning, Klassificeringsalgoritmer, Ljudinspelning, Maskininlärning, Akustisk signalbehandling 


\section{Contents}

List of Acronyms and Abbreviations . . . . . . . . . . . . v

1 Introduction 1

1.1 Problem . . . . . . . . . . . . . . . . . . 2

1.2 Objective and Contributions $\ldots \ldots \ldots \ldots$

1.2 .1 Delimitations . . . . . . . . . . . . . . . . . . . . 3

1.3 Research Methodology $\ldots \ldots$. . . . . . . . . . . . . 4

1.4 Outline . . . . . . . . . . . . . . . . . . . . 4

\begin{tabular}{|lll}
2 & Background & 5
\end{tabular}

2.1 Classification Algorithms . . . . . . . . . . . . . 5

2.1 .1 Hidden Markov Model . . . . . . . . . . . . . 6

2.1.2 Gaussian Mixture Model and GMM-HMM . . . . . 7

2.1 .3 GenHMM . . . . . . . . . . . . . . . . . . 8

2.1 .4 Long Short-term Memory . . . . . . . . . . . . . . 10

2.1 .5 Convolutional Neural Network . . . . . . . . . . . . 12

2.1 .6 Support Vector Machine . . . . . . . . . . . . . . 13

2.2 Mel-frequency Cepstral Coefficients . . . . . . . . . . . . . 14

2.3 Related Work . . . . . . . . . . . . . . . . . . . . 15

2.3.1 Audio Scene Analysis and Noise Classification . . . . 15

2.3 .2 Audio Classification . . . . . . . . . . . . . 18

2.3 .3 Audio Quality Assessment . . . . . . . . . . . . . . . 19

3 Methods 21

3.1 Single-label Study . . . . . . . . . . . . . . . . . . . 21

3.1 .1 Dataset Generation . . . . . . . . . . . . . . . 22

3.1 .2 Feature Extraction . . . . . . . . . . 23

3.1 .3 Algorithm Implementation . . . . . . . . . . . . . . 23

3.1 .4 Hyperparameter Search. . . . . . . . . . . . . . . . . . . . . . . . 25

3.2 Multi-label Study . . . . . . . . . . . . . . 25 
3.2.1 Adapted Dataset Generation . . . . . . . . . . . . 27

3.2.2 Classification . . . . . . . . . . . . . . . 28

3.2 .3 Tailored Features . . . . . . . . . . . . . . . 30

3.3 Experiments and Evaluation . . . . . . . . . . . 31

3.3.1 Confusion and Specificity Experiment . . . . . . . . 31

4 Results

$4.1 \quad$ Single-label Comparison Study . . . . . . . . . . . . . . . . 33

4.2 Multi-label Comparison Study . . . . . . . . . . . . . . . . . . . . . . . 34

$4.2 .1 \quad$ Optimal Feature and Classifier . . . . . . . . . . . . . 34

$4.2 .2 \quad$ Voice Activity Detection . . . . . . . . . . . . . . . . . . . . . . . . 39

4.2 .3 Ensemble Classification . . . . . . . . . . . . . . . . 39

4.2 .4 Confusion and Specificity Experiment . . . . . . . . . . . . . . . . . . . . 44

4.3 Reliability and Validity . . . . . . . . . . . . . . . 44

5 Discussion and Conclusions 45

$5.1 \quad$ Discussion . . . . . . . . . . . . . . . . . . . 45

5.2 Sustainability and Ethics . . . . . . . . . . . . . . . . . . . . . . . . . . . . 48

5.3 Limitations . . . . . . . . . . . . . . . . . 48

5.4 Future work $\ldots \ldots \ldots \ldots$

5.5 Conclusions $\ldots \ldots \ldots \ldots \ldots$

\begin{tabular}{ll}
\hline Bibliography & 51
\end{tabular}

\begin{tabular}{ll}
\hline Documentation & 57
\end{tabular} 


\section{List of Acronyms and Abbreviations}

ADT Audio Degradation Toolbox

CNN Convolutional Neural Network

DCT Discrete Cosine Transform

EM Expectation Maximization

FC Fully Connected

FFT Fast Fourier Transform

GenHMM Generative Model based HMM

GMM Gaussian Mixture Model

GMM-HMM GMM based HMM

HMM Hidden Markov Model

ITU-T ITU Telecommunication Standardization Sector

k-NN $k$-Nearest Neighbors

LPC Linear Predictive Coding

LSF Line Spectral Frequencies

LSTM Long Short-term Memory

MFCC Mel-frequency Cepstral Coefficient

MLP Multi-layer Perceptron

NLL Negative Log Likelihood

RBF-NN Radial Basis Functions Neural Network

ReLU Rectified Linear Unit

RMS Root Mean Square 
vi|List of Acronyms and Abbreviations

RNN Recurrent Neural Network

SNR Signal-to-noise Ratio

STFT Short-time Fourier Transform

SVM Support Vector Machine

VAD Voice Activity Detector 


\section{Chapter 1}

\section{Introduction}

In speech therapy and in phoniatrics there is often a need to record a patient's voice, either for research purposes, later analysis or consulting colleagues in difficult cases. As with all measurements there is a risk of introducing noise in the recording. Disturbances may arise from several different sources: electric hum caused by the power grid, reflections from the floor or furniture leading to reverberation in the signal, and environmental noise from the air-conditioning are just a few examples. As the human voice has a large dynamic range certain measurements need a very good recording environment to provide meaningful data [1, sec. 5.3], [2].

In 1995, Gelfer and Fendel [3] compared the quality of digitally stored recordings to that of tape recordings and found that parameters used to evaluate the voice differ depending on the type of recording. While the differences were deemed likely too small to affect clinical judgements they noted that some parameters would be unreliable when determining the effectiveness of therapy if computed from tape recordings. Even if using only digital recordings these problems may persist as different hardware and software configurations may respond differently to fan noise, causing noticeable effects in the measured vocal parameters [4]. In a more modern setting, Barsties and De Bodt [5] provide an overview of hardware, software, and environmental factors that must be taken into account when recording for voice quality assessment.

Consequently, we must not only consider the recording environment but also look for artefacts that may be caused by the recording setup itself. While experienced technicians are able to troubleshoot and eliminate potential issues, the equipment and expertise needed to detect subtle disturbances are not always readily available at smaller clinics. Thus we turn to automated methods for detection of signal corruptions. 
Speech recognition and speech enhancement are prominent fields in which machine learning and audio signal processing are combined. The robustness of methods is often tested by, for example, adding background noise to a signal at different Signal-to-noise Ratios (SNRs) [6], [7]. There are other, more relevant fields which primarily take an interest in signals with either pure noise or both speech and noise content, such as Auditory Scene Recognition and Noise Classification. Also relevant are certain methods for assessing the quality of an audio recording. Section 2.3 provides an overview of related work in these fields, while the rest of the current chapter describes this study's problem statement, objective and delimitations.

\subsection{Problem}

The problem we wish to solve regards discovering potential degradations in a recording of human speech, without the need for manual inspection by a trained expert. This study will focus on the following research questions:

1. Which machine learning algorithms are the most suitable for detecting selected signal corruptions in speech recordings? The results likely differ depending on the specific corruption investigated, so both overall and case-by-case performance is of interest. Though only a limited number of corruptions can be tested in this study we hope to find algorithms which can be expected to generalize well to new types of signal issues.

2. How viable is the machine learning approach for detection of these signal corruptions? The algorithms in point 1 must perform well enough that they are meaningful to use. In practice, this means they should reliably provide a classification accuracy much higher than chance and minimize the confusion between different corruptions. Various methods to increase the performance of the algorithms are also investigated, both on a general basis and for specific corruptions.

While not a research question in itself, we also have to solve the problem of how to evaluate our algorithms. Real testing data can be hard to come by as recordings of poor quality are unlikely to be saved and labeled, and constructing a corpus from scratch is out of scope for this study. While there are datasets with noisy speech, these are often constructed with telephony or speech recognition in mind and may focus on corruptions we are not interested 
in. Thus we must consider how to construct an artificial dataset from an existing corpus for our purposes.

\subsection{Objective and Contributions}

The purpose of this project is to create a prototype of a tool which can help speech therapists ensure their recordings are of good quality. If successful such a tool could help reduce the number of re-recordings necessary, which would mean fewer visits to the clinic for patients and less work for the clinicians, and maybe also increase the quality of measurements used for other purposes than diagnostics (such as research). Additionally, our dataset construction may be useful for robustness testing in other research fields as we may devise new corruption types which are otherwise rarely used.

To achieve this we create a dataset construction tool where different corruptions can be easily defined and a test bed where we can change the algorithm and feature modularly. We also implement or create interfaces for the algorithms we choose to evaluate. The tools developed for these purposes are made available so that others may continue on the work. Thus, ease of use and proper documentation are another aspects to consider.

\subsubsection{Delimitations}

As previously mentioned, the resources needed to record enough data for our purposes are not available at this time, and properly labeling a sizeable dataset would also be out of scope. Further, we will make no attempt at rectifying the defects which are identified by the tool, as one might in a speech enhancement algorithm, but merely alert the user to the problems found.

We limit the corruptions to a select few: three types of audible additive noise, random muting of the audio signal, infrasound, and hard and soft clipping. To apply these we need no contextual information about the speech content, which would have been needed to retroactively apply e.g. puff sounds at plosives. As for the source of the speech signal we look for suitable speech corpora. These recordings are characterized by phonetically diverse sentences spoken by (mostly) healthy individuals, and differ from the exercises sometimes used by speech therapists which for example include held vowels or singing. 


\subsection{Research Methodology}

First, we perform a literature study to identify five algorithms to test. We then perform a small scale experiment with strong assumptions: each recording is only affected by at most one corruption, the only corruptions possible are various types of additive noise, and only one type of feature is tested. The algorithms which perform consistently well (based on classification accuracy, variance thereof, and training and classification time) are selected for the next round of experiments which includes multi-label samples, more corruption types, ensemble classification, preprocessing using a Voice Activity Detector (VAD), and other types of features.

\subsection{Outline}

Chapter 2 presents the background for the different classification algorithms used and approaches in related fields. Chapter 3 presents the methodology and method used in this study, which is split into a single-label and a multilabel part. The results of the experiments are presented in chapter 4 , and finally discussed in chapter 5. Finally, documentation for the developed tool is available in the appendix. 


\section{Chapter 2}

\section{Background}

In this chapter a brief introduction to the necessary theoretical background is provided. First, the classification algorithms used in this report are outlined. Second, the features used in the classifiers are described. Last, an overview of related works is given and their relevance discussed.

\subsection{Classification Algorithms}

Classification is the task of determining the group (class) to which a certain object (sample) belongs. For example, in speech recognition the sample may be a short waveform recording while the classes are a dictionary of possible words. In a binary classification task we only have two classes while in a multiclass setting there may be more. There is also a distinction between singlelabel and multi-label classification, where a sample may belong to multiple classes in the latter case. If we consider single-label classification the objective is formally to find the class $c$ of a sample $x$. This is often done by calculating

$$
c=\underset{c_{i}}{\arg \max } p\left(c_{i} \mid x\right) .
$$

Model-based classification approaches can use either generative or discriminative models. In the generative case we create a model for $p\left(x \mid c_{i}\right)$ and $p\left(c_{i}\right)$, from which eq. 2.1 can be computed using Bayes' rule. A side effect of modeling these two probabilities is that we can use our model to generate new samples which are similar to the samples in the training set. In the discriminative case we instead create a model for $p\left(c_{i} \mid x\right)$ directly [8, sec. 4.3].

In this section the five classification algorithms used in the study are described. Of these, the Gaussian Mixture Model based Hidden Markov 
Model (GMM-HMM) and Generative Model based Hidden Markov Model (GenHMM) are generative models, while the Long Short-term Memory (LSTM), Convolutional Neural Network (CNN) and Support Vector Machine (SVM) implementations can be considered discriminative models. As described in section 2.3, there are other algorithms (such as $k$-Nearest Neighbors or Multi-layer Perceptron) or variants of the algorithms used (such as Gated Recurrent Units) with widespread usage that could also have been interesting in this study. We limited the number of algorithms to five to keep the implementation time reasonable and to be able to study the chosen algorithms more thoroughly.

The algorithms were selected both to give a mixture of state-of-the-art machine learning methods and traditional signal processing methods and to be relatively easy to implement. The GMM-HMM has been successfully used in a broad range of similar problems, and the related GenHMM has shown promising results in speech recognition [7]. Recurrent Neural Networks such as the LSTM are also designed to handle sequential data and make for an interesting comparison. CNNs are used in audio event detection and classification (see section 2.3.2), and should also have the potential to perform well in our setting. Lastly, the SVM is chosen as an established, straightforward classifier to provide another alternative to the many neural network algorithms.

\subsubsection{Hidden Markov Model}

The Hidden Markov Model (HMM) is a common model for sequences of vectors which vary over time. Assume that we have vector sequence $X=$ $\left\{\mathbf{x}_{1}, \ldots, \mathbf{x}_{T}\right\}, \mathbf{x}_{t} \in \mathbb{R}^{N}$. One simple way of modeling such a series would be to let each $\mathbf{x}_{t}$ be a sample of single random variable $\mathbf{X} \sim p(\mathbf{x} \mid \theta) \forall t$, where $\theta$ are the parameters of the distribution. The drawback is that in this case we would be unable to model any type of time dependence. In an HMM we instead let $\theta$ depend on a hidden state $z_{t}$ and thus have the output probability distribution

$$
\mathbf{X}_{t} \sim p\left(\mathbf{x} \mid z_{t}\right),
$$

the distribution which is sampled from at a given time instant.

The hidden state is a random, discrete variable which may assume one of $S$ different values. Depending on the sequence being modeled, $X$, we adapt our choice of output probability distribution. Gaussian distributions and Gaussian Mixture Models (GMMs) are common choices for continuous sequences, the latter of which is described below, but a discrete distribution may also be useful 
in conjunction with a clustering algorithm or Vector Quantization [9]. We have one distribution for each possible state, each with its own parameters $\theta_{s}$. Using Gaussian distributions this would result in $S$ different mean vectors $\mu_{s}$ and covariance matrices $\Sigma_{s}$.

To generate samples from an HMM we begin by choosing a random initial hidden state $z_{1}$ according to a distribution $\pi$. The first sample $\mathbf{x}_{1}$ is then drawn from

$$
\mathbf{X}_{1} \sim p\left(\mathbf{x} \mid z_{1}\right)
$$

after which we choose our next state $z_{2}$. This is done via a transition matrix $A$ such that

$$
P\left(z_{2} \mid z_{1}\right)=A_{z_{1}, z_{2}},
$$

after which we sample $\mathbf{x}_{2}$ and choose $z_{3}$, and so on. Thus, the parameters which make up the HMM are the initial state distribution $\pi$, the transition matrix $A$, and the parameters of each output distribution $\theta_{s}$. Training is done via the Baum-Welch algorithm, also known as the Forward-backward algorithm, which is a special case of Expectation Maximization (EM) [8, sec. 13.2].

There are different terms for HMMs with specific types of transition matrices. In a left-to-right $\mathrm{HMM} A$ is upper triangular, and in an ergodic HMM all elements in $A$ are nonzero.

\subsubsection{Gaussian Mixture Model and GMM-HMM}

The Gaussian Mixture Model (GMM) is commonly used to model or approximate probability distributions. It consists of a linear combination of $K$ different Gaussian distributions which can be written

$$
p(\mathbf{x})=\sum_{k=1}^{K} \pi_{k} \mathcal{N}\left(\mathbf{x} \mid \mu_{k}, \Sigma_{k}\right),
$$

where $\pi_{k}, \mu_{k}$ and $\Sigma_{k}$ are the weight, mean vector and covariance matrix, respectively, of each mixture component. Importantly, the weights $\pi_{k}$ satisfy $\sum_{k} \pi_{k}=1$ and $\pi_{k} \geq 0 \forall k$, so $\pi$ forms its own discrete probability distribution. Thus when we sample a value $\mathrm{x}$ from the GMM, we can intuitively understand it as first picking a component $z$ (a sample of $Z \sim \pi$ ) and then sampling $\mathrm{x}$ from that component using

$$
\mathbf{X} \sim p(\mathbf{x} \mid z)=\mathcal{N}\left(\mathbf{x} \mid \mu_{z}, \Sigma_{z}\right)
$$


Like HMMs, GMMs are trained using Expectation Maximization [8, sec. 9.2].

A GMM can be used on its own or as the output probability distribution in an HMM, the latter of which is referred to as a GMM-HMM, In the former case we cannot directly model any time dependence but it may still be useful for signal processing purposes. For example, Maijala et al. [10] develop a noise monitoring system based on GMM classifiers and Kotropoulos and Samaras [11] use GMMs as a preprocessing step, using the learned mean vector and covariance matrix diagonal (dubbed "Gaussian supervector") as a feature. See section 2.3.1 for further details.

The choice of covariance matrices $\Sigma_{k}$ depends on the features in question. If Mel-frequency Cepstral Coefficients (MFCCs) are used, which are described in section 2.2, diagonal matrices may suffice as the individual coefficients are uncorrelated [12]. The more elements of $\Sigma_{k}$ which are allowed to be nonzero, and the higher the number of mixture components $K$, the more data is needed during training of the model.

\subsubsection{GenHMM}

The Generative Model based HMM GenHMM, or Generator-mixed HMM, is another variant of HMM proposed by Liu et al. [7]. They are similar to GMM-HMMs, but instead of using Gaussian distributions as mixture components they use a special type of neural networks called generators. The generators are designed to be trainable in the EM framework. Each combination $s, k$ of state $s$ and component $k$ has its own probability distribution which is realized by transforming a standard normal distribution.

The steps to perform the generator transform are as follows:

1. Let $\mathbf{z}$ from $\mathbf{Z} \sim \mathcal{N}(0, I)$ be a sample from the multivariate standard normal distribution in $\mathbb{R}^{D}$, where $D$ is even.

2. Partition $\mathbf{z}$ into two parts, $\mathbf{z}^{(a)}$ and $\mathbf{z}^{(b)}$. This is done using a mask, for example

$$
\left\{\begin{array} { l } 
{ z _ { i } ^ { ( a ) } = z _ { 2 i - 1 } } \\
{ z _ { i } ^ { ( b ) } = z _ { 2 i } }
\end{array} \quad \text { or } \left\{\begin{array}{l}
z_{i}^{(a)}=z_{i} \\
z_{i}^{(b)}=z_{D / 2+i}
\end{array} \quad, i=1, \ldots, \frac{D}{2} .\right.\right.
$$

3. Use $\mathbf{z}^{(a)}$ as input into a neural network to obtain scale and shift factors. In our case the network consists of two hidden layers of dimension $H$ with Leaky ReLU activations and an output layer of dimension $2\left|\mathbf{z}^{(b)}\right|$. Denote the parameters of this network $\theta^{(b)}$. The output is split into $\mathbf{s}^{(b)}$ 
and $\mathbf{t}^{(b)}$, where $\mathbf{s}^{(b)}$ contains the first $\left|\mathbf{z}^{(b)}\right|$ elements and $\mathbf{t}^{(b)}$ the last, and used to calculate

$$
\mathbf{z}^{\prime(b)}=\left(\mathbf{z}^{(b)}+\mathbf{t}^{(b)}\right) \odot \exp \left(\mathbf{s}^{(b)}\right)
$$

where $\odot$ denotes element-wise multiplication.

4. Use $\mathbf{z}^{\prime(b)}$ as input into another neural network with the same architecture and obtain the factors $\mathbf{s}^{(a)}, \mathbf{t}^{(a)}$. Correspondingly, this network has parameters $\theta^{(a)}$. Transform $\mathbf{z}^{(a)}$ into $\mathbf{z}^{\prime(a)}$ by similar application of eq. 2.3 .

5. Merge $\mathbf{z}^{\prime(a)}$ and $\mathbf{z}^{\prime(b)}$ to obtain $\mathbf{z}^{\prime}$ by applying the same mask as in eq. 2.2. We can summarize steps 2,5 as a single transform $\mathbf{z}^{\prime}=g(\mathbf{z})$, which is referred to as a coupling layer. These are described in more detail by Dinh et al. [13], and the computation graph is illustrated in figure 2.1.

6. Combine $n_{\text {chain }}$ coupling layers, each with unique neural network parameters, to obtain

$$
\mathbf{x}=\left(g_{1} \circ g_{2} \cdots \circ g_{n_{\text {chain }}}\right)(\mathbf{z}),
$$

where $\circ$ denotes function composition. The result is referred to as a generator.

Like with the GMM the goal of a generator is to model a complex distribution based on simpler, Gaussian distributions of latent variables.

For classification tasks we do not need to sample from the GenHMM in this manner. Instead we use the inverse transform

$$
\mathbf{z}=\left(g_{n_{\text {chain }}}^{-1} \circ \cdots \circ g_{1}^{-1}\right)(\mathbf{x}),
$$

which is possible as each step is invertible. For example, to obtain $\mathbf{z}^{(a)}$ from $\mathbf{z}^{\prime(a)}, \mathbf{z}^{\prime(b)}$ we compute $\mathbf{s}^{(a)}, \mathbf{t}^{(a)}$ using the neural network in step 4 and apply

$$
\mathbf{z}^{(a)}=\mathbf{z}^{(a)} \odot \exp \left(-\mathbf{s}^{(a)}\right)-\mathbf{t}^{(a)} .
$$

As stated above, training is done via Expectation Maximization.

The parameters we train in the GenHMM are both the regular HMM parameters and the neural network parameters $\theta$ in each coupling layer. As we increase the number of parameters, however, we also increase the amount of data needed to properly train the classifier. As early as in 1995 Morgan and Bourlard [14] noted that hybrid HMM-neural network models require more 


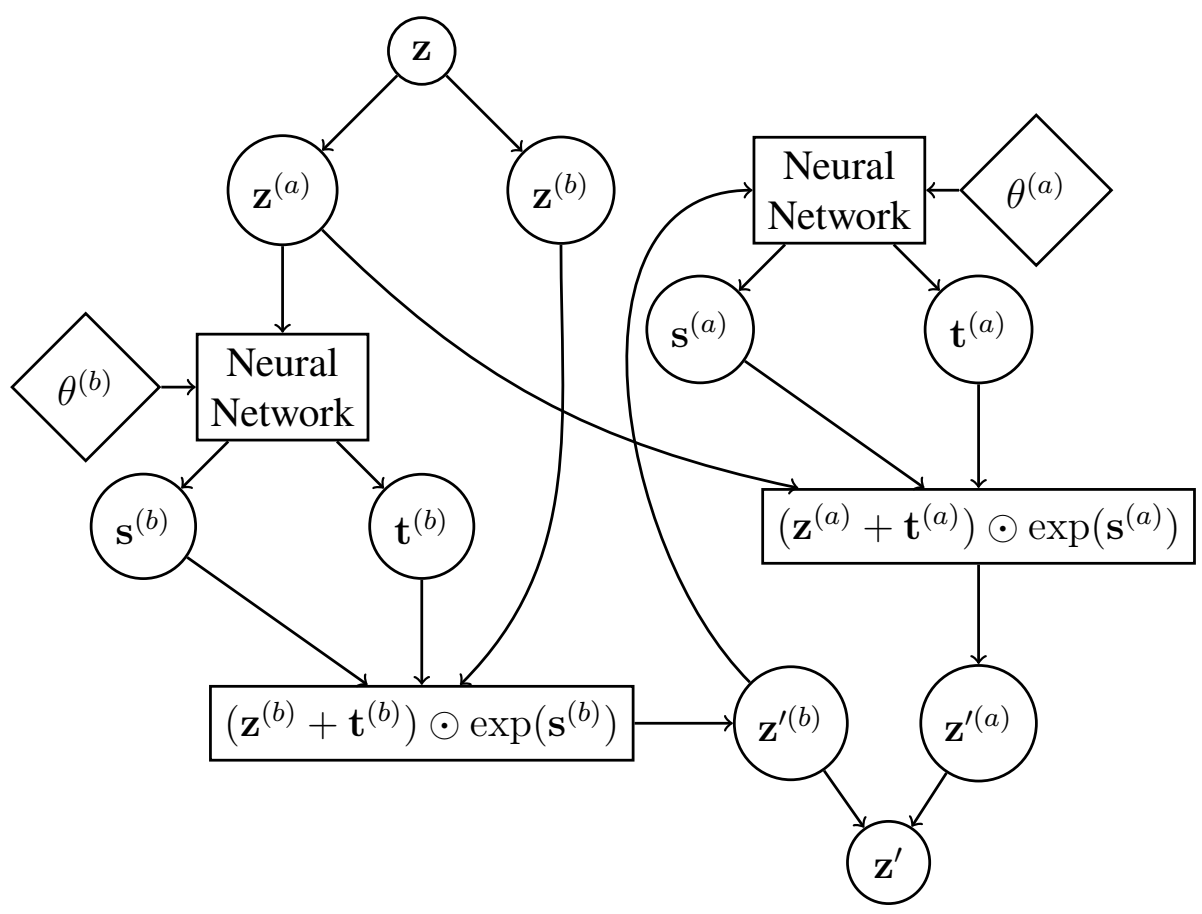

Figure 2.1: A graph showing the computation of a coupling layer $\mathbf{z}^{\prime}=g(\mathbf{z})$, steps 2.5. During training, the parameters $\theta^{(a)}$ and $\theta^{(b)}$ are learned.

data and computing power than discrete HMMs, which may be a limitation in our case as the data available is somewhat restricted (see section 3.1.1 on dataset generation).

\subsubsection{Long Short-term Memory}

Recurrent Neural Networks (RNNs) is a group of neural network architectures which includes Long Short-term Memory (LSTM) and which bears some resemblance to HMMs. Notably, $\overline{\mathrm{RNN}}$ layers can process variable-length input. Classification using an RNN begins with the initialization of a hidden state $\mathbf{h}_{0}$, after which the first input vector $\mathbf{x}_{1}$ is incorporated into the state and an output vector $\mathbf{o}_{1}$ yielded. This is done using functions $f, g$, the parameters of which are learned during training. The updated hidden state $\mathbf{h}_{1}=f\left(\mathbf{h}_{0}, \mathbf{x}_{1}\right)$ is then combined with the next input vector and the process is repeated until the input sequence ends. An example of $f$ is

$$
f\left(\mathbf{h}_{t-1}, \mathbf{x}_{t}\right)=\tanh \left(W_{x} \mathbf{x}_{t}+W_{h} \mathbf{h}_{t-1}+\mathbf{b}\right),
$$


where $W_{x}, W_{h}, \mathbf{b}$ are the learned parameters [15, entry RNN]. One can then either use the sequence of output vectors $O=\left\{\mathbf{o}_{1}, \ldots, \mathbf{o}_{n}\right\}$ as input into another RNN layer, or use the last output vector $\mathbf{o}_{n}$ as input into e.g. a Fully Connected (FC) layer to obtain a label.

In an LSTM, instead of having only a hidden state, a memory cell $\mathbf{c}$ is introduced by Hochreiter and Schmidhuber [16] to capture long-term contexts and alleviate the problem of vanishing or exploding gradients during training. To manage the information flow to and from the memory cells an input gate, forget gate, and output gate are also introduced, which are functions whose parameters we train. While the network topology in the original definition is flexible, in this section we use the activation functions and structure in the PyTorch [15] LSTM]layer implementation.

The input gate determines what new information, computed from $\mathbf{x}_{t}$ and $\mathbf{h}_{t-1}$, is added to the memory cell $\mathbf{c}_{t}$. It is written as

$$
\mathbf{i}_{t}=\sigma\left(W_{x i} \mathbf{x}_{t}+W_{h i} \mathbf{h}_{t-1}+\mathbf{b}_{i}\right),
$$

where $\sigma$ is the sigmoid function. The forget gate determines what information from the memory cell is kept, i.e. added from $\mathbf{c}_{t-1}$ to $\mathbf{c}_{t}$, and can be written as

$$
\mathbf{f}_{t}=\sigma\left(W_{x f} \mathbf{x}_{t}+W_{h f} \mathbf{h}_{t-1}+\mathbf{b}_{f}\right)
$$

The memory cell is updated via

$$
\mathbf{c}_{t}=\mathbf{f}_{t} \odot \mathbf{c}_{t-1}+\mathbf{i}_{t} \odot \tanh \left(W_{x c} \mathbf{x}_{t}+W_{h c} \mathbf{h}_{t-1}+\mathbf{b}_{c}\right),
$$

where $\odot$ again denotes the element-wise product. The output gate decides what information from the newly computed $\mathbf{c}_{t}$ is included in the new hidden state, $\mathbf{h}_{t}$. It is computed as

$$
\mathbf{o}_{t}=\sigma\left(W_{x o} \mathbf{x}_{t}+W_{h o} \mathbf{h}_{t-1}+\mathbf{b}_{o}\right)
$$

and the updated hidden state becomes

$$
\mathbf{h}_{t}=\mathbf{o}_{t} \odot \tanh \mathbf{c}_{t}
$$

Again, the matrices $W$ and vectors $\mathbf{b}$ are the parameters of the network which are learned during training, and the last output vector can be used with FC layers for classification [15, entry LSTM]. 


\subsubsection{Convolutional Neural Network}

Convolutional Neural Networks (CNNs) are named after the layers in which a convolution is applied between the input and one or more filters. They are commonly used in machine learning for image data, but they are also useful for sequences of vectors as the convolutional layer supports variable-length input. Interestingly, knowledge from CNNs pretrained on image recognition can be transferred into audio classification CNNs to improve their performance [17].

A textbook implementation of a CNN looks as follows: assume that we have an $N_{X} \times M_{X}$ sample $X$ and $n_{\text {filters }}$ different $N_{f} \times M_{f}$ matrices $F^{(1)}, \ldots, F^{\left(n_{\text {filters }}\right)}$ which will act as our filters. In a convolutional layer we convolve each of the filters with our input $X$ and output the result as an $N_{o} \times M_{o} \times n_{\text {filters }}$ tensor $R[18]$. That is, we compute

$$
R_{i j k}=\sum_{n=0}^{N_{f}-1} \sum_{m=0}^{M_{f}-1} X_{(i+n),(j+m)} F_{(n+1),(m+1)}^{(k)}
$$

for $i=1, \ldots, N_{X}-N_{f}+1, j=1, \ldots, M_{X}-M_{f}+1$, and $k=$ $1, \ldots, n_{\text {filters. }}$ The trainable parameters in a convolutional layer are the filter matrices $F^{(1)}, \ldots, F^{\left(n_{\text {filters }}\right)}$.

Eq. 2.5 extends naturally to input tensors $X$ of orders 3 or 1 if the filters are given the same order. A stride can be applied during the convolution, in which case we only keep the elements of $R$ where $i \equiv 1 \bmod s_{i}$ and $j \equiv 1 \bmod s_{j}$. This is commonly used in conjunction with zero-padding of $X$ to make sure no column or row is missed. The output $R$ is passed through an activation function, such as Rectified Linear Unit (ReLU), and can then be used with e.g. a Fully Connected layer and a softmax function to yield classification probabilities [17].

Pooling operations are often used together with convolutional layers to reduce the amount of computations and parameters needed while still maintaining the most important data [18]. If we apply a max-pooling operation to $R$ defined above we first need to select a chunk size $c=\left(c_{1}, c_{2}, c_{3}\right)$. We then split $R$ into regions of size $c$ and compute the maximum element in each region, outputting the result in a $\frac{N_{o}}{c_{1}} \times \frac{M_{o}}{c_{2}} \times \frac{n_{\text {filters }}}{c_{3}}$ tensor $R^{\prime}$.

If we classify a variable-length sequence of vectors we cannot simply add an FC layer after the convolutional layer, as the FClayer only supports input of a constant length. One way to circumvent this is to split the sequence into fixed-length segments and use each segment for training or classification separately, similarly to Lee et al. [19]. Another solution is to use a recurrent 
layer after the convolutional layer, as done by Cakir et al. [18].

\subsubsection{Support Vector Machine}

Classification in a Support Vector Machine (SVM) is done by finding a hyperplane that separates the samples of two different classes [8, sec. 7.1]. Because such a boundary may be hard to find in low-dimensional data the samples are generally projected into a higher dimension, and a separating hyperplane is found in the higher dimension instead. Mathematically, this process can be simplified by the so-called kernel trick, in which we replace the similarity metric $\mathcal{K}(\mathbf{x}, \mathbf{y})$ between samples while keeping the samples in the low-dimensional representation [8, ch. 6]. A common choice of such a kernel is the radial basis function,

$$
\mathcal{K}(\mathbf{x}, \mathbf{y})=\exp \left(-\gamma|\mathbf{x}-\mathbf{y}|^{2}\right)
$$

There may be several similar hyperplanes that separate the classes, and the most interesting of these is the hyperplane which has the largest margin between the two classes. Consider the case with $N$ samples $\mathbf{x}^{(1)}, \ldots, \mathbf{x}^{(N)}$ with associated class labels $t_{1}, \ldots, t_{N} \in\{-1,1\}$. Training an SVM is then equivalent to maximizing

$$
\sum_{i=1}^{N} \alpha_{i}-\frac{1}{2} \sum_{i=1}^{N} \sum_{j=1}^{N} \alpha_{i} \alpha_{j} t_{i} t_{j} \mathcal{K}\left(\mathbf{x}^{(i)}, \mathbf{x}^{(j)}\right)
$$

with regards to $\alpha$, under the constraints $\sum_{i} \alpha_{i} t_{i}=0$ and $\alpha_{i} \geq 0 \forall i$. A sample $\mathbf{x}^{(i)}$ for which $\alpha_{i}>0$ is referred to as a support vector. Once the $\alpha$ are found we can classify a new sample $\mathbf{x}^{\prime}$ using the sign of the indicator function

$$
y\left(\mathbf{x}^{\prime}\right)=\sum_{i=1}^{N} \alpha_{i} t_{i} \mathcal{K}\left(\mathbf{x}^{(i)}, \mathbf{x}^{\prime}\right)+b,
$$

where $b$ is a threshold parameter which can be computed using any support vector via $t_{i} y\left(x^{(i)}\right)=1$. Note that only support vectors are needed to evaluate $y\left(\mathbf{x}^{\prime}\right)$ as the other samples have $\alpha=0$ by definition [8, sec. 7.1].

In cases with data that is not completely separated (which is especially likely for large $N$ ) there may not be a separating hyperplane even in the projected dimension. One solution to this is to introduce slack by allowing support vectors to lie inside the margin to the hyperplane. The number of vectors allowed and how far beyond the margin they may lie are controlled by 
the regularization parameter $C$; increasing it provides more slack, and setting it to 0 is equivalent to not introducing slack. A side effect of increasing $C$ is that the number of support vectors increases, which in turn increases training and evaluation time [20], [8, sec. 7.1.1].

Using the common kernels, SVMs are not capable of handling vectors of different lengths or, by extension, sequences of vectors. We thus apply the same approach as with the $\overline{\mathrm{CNN}}$ and split our data into frames. Another consideration is that the SVM in this formulation only works with binary classification. If we have $n>2$ different classes we in general resort to training either $\frac{n(n-1)}{2} 1$-versus- 1 classifiers or $n 1$-versus-rest classifiers, but there are other approaches to multi-class SVM classification [8, sec. 7.1.3].

\subsection{Mel-frequency Cepstral Coefficients}

Mel-frequency Cepstral Coefficients (MFCCs) were designed in the 1980's and are today one of the most common features used for various type of audio classification and quality assessment tasks. They are designed to mimic human hearing so that two things that sound alike also are close in the MFCCdomain [21]. To compute MFCCs one performs a Short-time Fourier Transform (STFT) and divides the resulting spectrogram along the frequency axis using a filter bank. The filters are triangular, overlapping, and evenly spaced according to the mel scale, which is a logarithmic scale based on perceptual experiments designed to create equal pitch increments. It is common to use around 20 filters, and figure 2.2 shows an example of a filterbank with 23 filters. The energy in each filter is then computed to yield a mel-frequency spectrogram [22, sec. 5.6.3].

The (log-)mel-frequency spectrogram can be used as a feature itself [23], but in our case we are interested in cepstral coefficients. Cepstral (derived from spectral) is a term to describe spectra of spectra and to compute the coefficients we take the Discrete Cosine Transform (DCT) of our melfrequency spectrogram. The number of points in the DCT is chosen so that it is fewer than the number of filters, and 13 is a common choice. The resulting vector sequence of DCT coefficients are the MFCCs, a compact representation of the shape of the spectrum at a given time instant. It is not uncommon to combine MFCCs with MFCC- $\Delta$ or MFCC- $\Delta \Delta$, which correspond to the first and second order time difference, respectively. 


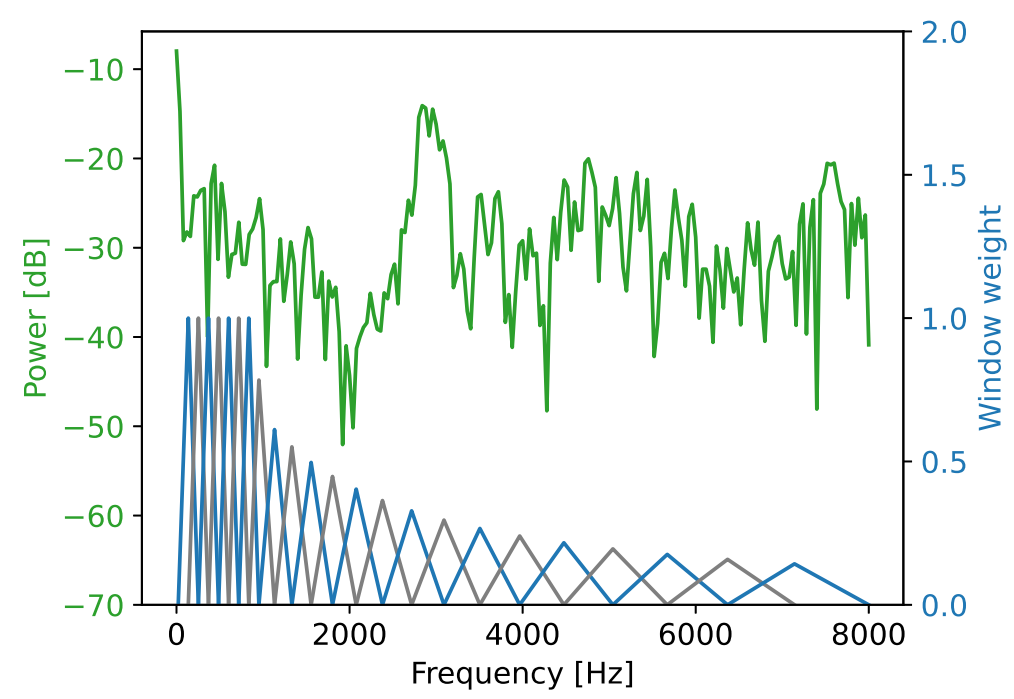

Figure 2.2: 23 mel-spaced filters overlaid on a spectrum. Note that for frequencies below $1000 \mathrm{~Hz}$ the filters are evenly spaced according to the regular hertz scale.

\subsection{Related Work}

The literature study mainly covered three related areas: audio scene analysis, noise classification and audio quality assessment. The field of audio classification, such as classification of general audio data, was also considered. Relevant papers, patents and books were located either through Google Scholar keyword searches, recommendations from supervisors, or through the citations of already-read papers.

\subsubsection{Audio Scene Analysis and Noise Classification}

Audio scene analysis and noise classification are two closely related fields, as audio scene analysis often involves classifying environmental background noise. Many different machine learning and pattern recognition methods have been attempted. As additive background noise is a very common type of noise that will need to be considered in our study, these methods are relevant for study to determine if they are suitable for our purposes.

Ma et al. [12] develop a system for context-awareness in mobile phone applications which is based on Gaussian HMMs using MFCC features. The authors record 80 samples each from ten different acoustic environments, for 
example a lecture hall and a football match, and train the classifiers to detect the environment in which a sample was recorded. The authors find that leftto-right HMMs outperformed ergodic HMMs, They introduce a confidence measure based on the log-likelihoods, $L_{1}>L_{2}$, for the two best matching scenes, formulated as

$$
\text { conf }=\left|\frac{L_{1}-L_{2}}{L_{1}}\right| .
$$

This can be used both to determine the estimated correctness of a classification, and to perform unsupervised learning of new acoustic environments or augment the existing environments.

Further, Ma et al. also perform sound event classification tests, and note that fewer HMM states are needed for this task. A hierarchical model is proposed where a recording can first be classified by environment-versus-event classifiers and then sorted into increasingly specific categories. An interesting takeaway from their discussion is that $100 \%$ accuracy may in some cases be impossible to achieve as certain categories in their database are heterogeneous in the MFCC domain. This means that the grouping of sounds is not based entirely on acoustic similarity but also on some additional context which is unavailable to the classifiers, such as the materials involved in a collision sound.

Couvreur et al. [9] use HMMs to classify noise events originating from five different types of vehicles. They use cepstral coefficients derived from Linear Predictive Coding (LPC) as their features, which, like the mel-frequency spectrum, is a technique for summarizing the spectral envelope a signal. The coefficients are made discrete via vector quantization so that discrete HMMs can be used. With a total of 184 noise recordings the dataset of vehicle noise is small in comparison to datasets used by other studies. Various LPC frame lengths and HMM state counts were tested and a peak accuracy of $95.3 \%$ was achieved, while human listeners achieved $91.8 \%$ accuracy in informal listening tests. The authors base most parameter choices on defaults used in speech recognition but note that longer analysis frames improved performance for their task.

Similarly, Maijala et al. [10] develop a noise monitoring system for classifying noise as background or target noise. In their experiments, target noise encompasses all sounds generated by the activity in a rock crushing plant, such as alarms and machines, while wind and outside traffic are considered background noise. Both a GMM and a Multi-layer Perceptron (MLP) are used together with MFCC (including $-\Delta$ and $-\Delta \Delta$ ) features, and they both performed equally well. Various feature settings were tested and 
the best results were achieved using $\mathrm{MFCC}+\Delta$ with 13 cepstral coefficients, using $100 \mathrm{~ms}$ analysis windows. The authors also conducted an informal study where the system was extended to monitor multiple target classes.

El-Maleh et al. [24] perform a series of classification tests on five types of background noise common in mobile telephony: babble, car, bus, factory, and street noise. They use Line Spectral Frequencies (LSFs) as features, which are a different representation of $\mathrm{LPC}$ coefficients that the authors found maximized the class separability. Four classification methods are tested: Quadratic Gaussian, Least-Square Linear, $k$-Nearest Neighbors (k-NN), and Decision Tree. The tests involve classification of the five noise types and distinguishing between the noise types and speech. They also perform experiments with new noise types (such as ambient restaurant noise) or multiple superimposed speech signals. Overall the quadratic Gaussian classifier gave the best results.

Peltonen et al. [25] have a similar problem formulation to Ma et al. [12] and a dataset with 226 recordings from 26 different scenes. In their study two separate classifier types, $\mathrm{k}-\mathrm{NN}$ and GMM, are trained with a wide range of different features in tests to determine the best feature combination and the test sequence length needed, among others. The tested features include zerocrossing rate, spectral centroid, MFCCs, LPCs and their cepstral coefficients, band-energy ratios, and variance and delta features of each type. The best performance was achieved with MFCCs for the GMM and with band-energy ratios for the $\mathrm{k}-\mathrm{NN}$ classifier.

Kotropoulos and Samaras [11] and Qi et al. [26] both try to identify the mobile phone by which a recording was made, which has applications in forensics. This is an interesting variation in that speech is included in the signal (unlike [9], [10], [12], [24], [25]) and that properties of the recording setup, not environmental noise, are the basis for classification. The two studies use different approaches. Kotropoulos and Samaras expect different frequency responses in the different devices. They extract 23-dimensional MFCCs from the recordings, fit a GMM for each recording, and use the GMM s mean vector and covariance matrix diagonal as the feature. Classification is done via SVMs with different kernels, a Radial Basis Functions Neural Network (RBF-NN), and an MLP, with the RBF-NN providing the best results. Qi et al. instead expect different additive background noise in different devices. They use a speech enhancement algorithm to get a de-noised signal, then subtract this from the original to get only the noise signal. A Fast Fourier Transform (FFT) histogram of the noise signal is computed and fed into one of three classifier types: a Softmax regression model, an MLP, or a CNN They successfully use an ensemble of voting classifiers to boost their classification accuracy. 
Like many others in telecom-related research, Leman and Faure [27] classify noise based on its dominant perceptual characteristic (e.g. crackling or hissing) rather than its source (such as traffic or wind). They use decision trees to find appropriate features and thresholds for these with the goal of noise reduction and the constraint of low processing requirements. The chosen features were variation in acoustic signal level and the spectral flux, though cepstral features were not considered.

Lastly, Abreu et al. [28] perform classification of additive background noise in speech signals but use a VAD to extract only the non-speech segments. They use a dual-tree complex wavelet transform to extract features and classification is done via the Negative Selection Algorithm, which is very similar to $\mathrm{k}-\mathrm{NN}$ albeit with lower memory requirements. Different affinity measures are tested and the results are compared with SVM, MLP, and decision tree classifiers. The authors consider their algorithm to be the best, but the lack of discussion about the results, simplicity of the MLP, and the omission of a hardware specification for the classification time tests raises questions about their conclusions.

\subsubsection{Audio Classification}

While noise classification is one field related to audio scene analysis, other fields such as classification of general audio may also provide valuable insights [25]. Li et al. [29] test a broad range of different features in a comparison study to classify continuous audio data. Their algorithm both detects the location of different segments and labels the segments with the appropriate class, such as music, speech, multiple speakers, and noisy speech. They conclude that the choice of features is important and that cepstral-based features such as MFCC are better suited than spectral (e.g. band-energy ratio) or temporal features (e.g. zero-crossing rate).

Similarly, Hussain and Haque [17] classify speech, noise and music using a new 1DCNN architecture. They use MFCC features and perform frame-wise classification of sequences as described in section 2.1.5 above. The results are compared with a GMM classifier, a Self-normalizing Neural Network, and an established 2D CNN. Their architecture yields comparatively good results and the authors demonstrate that knowledge from networks trained on image classification can be distilled and used to improve accuracy in audio classification.

Hershey et al. [23] perform audio classification on a very large scale, with a dataset consisting of 70 million videos with 30000 possible labels. They 
use log-mel spectrogram features and test various CNNs against a deep MLP baseline in one of few multi-label classification studies in this literature review. The authors find that the $\mathrm{CNN}$ architectures which have been successful in image recognition tasks perform well in the audio domain too, with minor modifications. Likewise, Lee et al. [19] show that CNN] architectures can be repurposed for other audio classification tasks than originally designed. Their approach to feature extraction is unique among the papers in this study in that they successfully use the raw waveform itself as input directly into their network.

Finally, Cakir et al. [18] compare a CNN RNN hybrid to other methods GMM MLP, CNN, and RNN for multi-label sound event detection. The proposed method uses frames of log-mel band energies which are input into a stack of convolutional layers, followed by a recurrent layer and a subsequent FC layer. The recurrent layer is a Gated Recurrent Unit, which is similar to the LSTM but has fewer parameters to train as there is no output gate. The CNN RNN was shown to outperform the other methods on most datasets, was more robust to pitch shifting the samples, and was consistent across all classes. However, its performance was heavily dependent on the amount of training data available.

\subsubsection{Audio Quality Assessment}

Audio quality assessment can be considered a related field to noise classification as feature extraction and overall model architectures are often similar. One difference is that noise classification tasks in general use "clean" data, where the speech signal (if at all present) has been removed during preprocessing either by a VAD or through some other means, while audio quality assessment by its nature requires a complete, degraded signal. This is sometimes accompanied by a clean reference signal (in an intrusive algorithm) or pseudoreference signal (in certain non-intrusive algorithms) [30]. The literature study focused on non-intrusive quality assessment algorithms.

Rix et al. [30] describe the history of speech quality assessment up until 2006. Early approaches in non-intrusive assessment were based on estimating the distance from a given signal to the space of ideal speech signals. In this problem formulation the choice of distance metric is important in itself. These methods were later augmented by HMM and GMM techniques, and vocal tract analysis to identify sounds and effects not caused by a human voice. However, since the article was written the field has evolved and modern machine learning algorithms are commonplace today. 
While a speech recognition algorithm is ideally robust to varying noise (in the acoustic sense), an audio quality assessment algorithm is ideally robust to the content of the signal itself, be that music [31] or speech [32], [33]. Ma et al. [12] note that a difference between speech recognition and acoustic environment classification is that assumptions can be made on the nature of speech (originating from a single source and with certain limitations), which one cannot in the latter case. A similar distinction is made in quality assessment and different algorithms exist for speech and for general sound.

ITU-T]recommendation P.563 [32] is a non-intrusive algorithm for speech quality assessment. Simplified, it performs vocal tract analysis to classify the speech as originating from a male, female, or "strongly roboticized" voice, and then proceeds to identify issues with the recording. It also detects additive noise, unnatural silence, and clipping, among others. The overall score is then based on what issues were identified in the analysis and their severity.

Avila et al. [33] uses the recommendation as a baseline for comparison with their method, and note that as it was developed to test speech codecs it is outperformed by other algorithms in reverberant speech. They make no attempt to identify specific signal issues, instead training a network to predict the mean opinion score that a human listener would assign a given sample. The same is true for Li et al. [31], who do not estimate the score directly but instead have users and classifiers rank differently degraded versions of the same content. 


\section{Chapter 3}

\section{Methods}

In this chapter the development of the dataset generation tool and classification test setup is described. First, the single-label study is described, including the implementation details of the five chosen algorithms. Next, the adaptations needed and functionality added in the multi-label study are presented. Finally, the evaluation method is described.

\subsection{Single-label Study}

In the single-label study [34] we consider three types of additive noise:

Electric Hum, which can occur due to ground loops in the recording setup. It can be described as a humming or buzzing sound with a 50 or $60 \mathrm{~Hz}$ fundamental tone depending on the frequency of the power grid.

Air-conditioning noise, which is caused by the ventilation system. It consists both of noise from fans and from the flow of the airstream itself.

White Noise, also known as static noise. It is characterized by having a flat power spectrum and is used here to represent the noise floor of a microphone, for instance.

We assume that any recording is affected by at most one of these noise types. Thus we have four classes in total as there is one for recordings without added noise (None).

Vogel and Morgan [2] describe additive noises as "a prominent cause for signal degradation." Notably, fan noise, noise caused by electrical effects such as poor insulation, and intrinsic noise in the recording devices are cited as 
common forms of additive noise. For our study, additive noises also have the benefit of being easy to simulate as the noise signal can simply be added to the clean signal. We use Signal-to-noise Ratios (SNRs) up to $40 \mathrm{~dB}$ as SNRs in excess of $42 \mathrm{~dB}$ have little effect on common forms of analysis [2], [5].

\subsubsection{Dataset Generation}

There are already some datasets with noisy speech such as NOIZEUS [6], with additive noise of different kinds, and TCD-VoIP [35], with degradations likely to occur during VoIP calls. However, these are most often used to test the quality of a speech recognition system or a codec and thus contain corruptions which are unlikely to be present in a clinical setting, such as restaurant babble noise. Further, we want to be able to expand on the types of corruptions and thus resort to generating our own dataset. This is done by applying effects to high-quality recordings to simulate a poor recording setup.

Similarly to Kotropoulos and Samaras [11] we base our dataset on the TIMIT corpus of speech recordings. It contains 2-8 s recordings of English speech by people with different American accents. Each recording is annotated with both the phonemes and the phrases being uttered, but this is not necessary for our needs. There are a total of 6300 audio files in 16-bit $16 \mathrm{kHz}$ mono NIST Sphere format. Each speaker has ten recorded utterances, which are designed to be phonetically diverse, and two of these utterances are spoken by every speaker.

We use recordings for the air-conditioning noise and electric hum as well, while the white noise is generated by the Audio Degradation Toolbox (ADT) [36]. Recordings were downloaded from Freesound [37] or collected by supervisor Sten Ternström and are available in the GitHub repository [38]. There are 15 air-conditioning recordings at a total of 12.5 minutes and 19 electric hum recordings at a total of 8.5 minutes. Where needed, the recordings are downsampled and their channels mixed together to comply with the TIMIT files.

Both the speech and noise recordings are randomly split into training and testing sets at approximately a 3:1 ratio. This means that a certain TIMIT speaker is likely to occur in both the training and testing set, as is a certain phrase, but never the same phrase uttered by the same speaker. Each utterance is assigned one of the four classes and, if Air-conditioning or Electric Hum is chosen, a random sample in that category is selected and added using the ADT. Training noise samples were selected for training utterances and likewise for testing, thus no recording appears in both the training and the testing set. For 
a certain dataset the $\underline{\mathrm{SNR}}$ is the same for all classes and utterances. After the noise is added the sample is normalized to avoid clipping and to ensure that an increased volume itself is not an indicator that noise is present.

Six different datasets, $A, B, B^{\prime}, C, D$ and $D^{\prime}$, are generated with slightly different settings. In datasets $A-C$ the noise was added at an SNR of $20 \mathrm{~dB}$ while in $D$ and $D$ ' the $\mathrm{SNR}$ was increased to $40 \mathrm{~dB}$ (making the noise less audible). All datasets except $A$ included a 1 s padding in the beginning and end of each utterance before the noise is added. As white noise was implemented later than the other classes only $C$ contains all four classes-the rest have only Electric Hum, Air-conditioning, and None. While the partitioning into training and testing sets is individually randomized for each dataset, all other parameters were the same for all datasets.

\subsubsection{Feature Extraction}

MFCCs are used by Liu et al. [7] in their GenHMM experiments. To ensure compatibility with their code we use the same tool, Kaldi [39], for feature extraction. The MFCCs are extracted with the default settings of computemfcc-feats, the most important of which are shown in table 3.1. One thing to note is that Kaldi by default applies Gaussian dither in the computation of MFCCs to provide a noise floor and avoid computing $\log 0$. This should, however, be orders of magnitude smaller than our applied noises and not affect the classification accuracy.

Table 3.1: Settings used for feature extraction in the single-label study.

\begin{tabular}{lrl} 
Parameter & Value & Note \\
\hline Frame length & $25 \mathrm{~ms}$ & \\
Frame shift & $10 \mathrm{~ms}$ & \\
Window type & Povey & Similar to Hamming \\
Frequency range & $20 \mathrm{~Hz}-8 \mathrm{kHz}$ & Lowest audible to Nyquist freq. \\
Number of bins & 23 & \\
Number of cepstra & 13 &
\end{tabular}

\subsubsection{Algorithm Implementation}

This section describes the specific parameters used for each classification algorithm. These were chosen based on a hyperparameter search detailed in section 3.1.4 
The GMM-HMM] is implemented by Liu et al. [7], whose implementation in turn is based upon the version in the Python module hmmlearn. The HMM has three states and the GMM has two mixture components with diagonal covariance matrices. The GenHMM implementation comes from the same authors and we again use three states and two probability components. There are a number of additional hyperparameters for the more complex GenHMM model and these are displayed in table 3.2. For both of the HMM variants we train one separate model for each class (including None) and classify based on the model with the highest emission probability of the observed feature sequence. The Fully Connected $(\overline{\mathrm{FC}})$ layers in the generators are trained using an adaptive learning rate with the Adam optimizer and Negative Log Likelihood (NLL) loss.

Table 3.2: Hyperparameters of the GenHMM

\begin{tabular}{lrl} 
Parameter & Value & Note \\
\hline$n_{\text {chain }}$ & 4 & \\
$H$ & 24 & Hidden dimension in FClayers \\
$p_{\text {drop }}$ & 0 & Dropout probability in FC layers \\
$D$ & 12 & Dimension of latent distribution \\
Mask type & cross & First case in eq. 2.2 \\
em_skip & 4 & \\
startprob_type & first & \\
transmat_type & triangular & Yields a left-to-right HMM
\end{tabular}

As the GenHMM is implemented in PyTorch we choose to implement the other neural network classifiers in PyTorch as well. The LSTM has two layers with a hidden dimension of 20 , followed by a fully connected $20 \times N_{\text {classes }}$ layer, and finally logarithmic softmax is applied to the output. The LSTM is also trained using the Adam optimizer and NLL loss, and uses a constant learning rate of 0.005 .

The idea for the CNN design is loosely based on that of Cakir et al. [18]. It operates on frames of 30 consecutive MFCC vectors concatenated into a single $30 \times 13$ matrix. As a frame shift of 30 is used, consecutive frames do not overlap. During training each frame is labeled the same as the full sequence it is extracted from, and during classification the mode of the labels assigned to a sequence's frames is used as the sequence label.

We have two convolutional layers in the $\mathrm{CNN}$, one with $1 \times 3$ channels and the other with $3 \times 6$ channels. Both use a $4 \times 3$ size kernel and a zeropadding of 2 in each dimension. After each convolutional layer we apply a 
ReLU-nonlinearity followed by a $3 \times 3$ max pooling operation. Afterwards, the channels are stacked and the entire vector is fed into a $\mathrm{FC}$ network with ReLU-activations, a single hidden layer of size 32 and an output dimension equal to $N_{\text {classes. }}$. As with the LSTM we finally apply logarithmic softmax to get the class probabilities. The $\overline{C N N}$ is trained using the Adam optimizer with a constant learning rate of 0.03 , using NLL loss.

For the SVM the features are preprocessed similarly to the CNN except we use 20 concatenated feature vectors and a frame shift of 15 vectors. Frames are normalized during training to have zero mean and unit variance, and the same learned scaling and shifting is applied during classification. The SVM classifier is a wrapper for the sklearn SVC implementation [20], and uses radial basis functions, a regularization parameter $C=1.0$, and a kernel coefficient $\gamma=\frac{1}{20 \cdot 13}=3.85 \cdot 10^{-3}$.

\subsubsection{Hyperparameter Search}

A hyperparameter search was conducted to find appropriate settings for each classifier. The starting points were the values used in the relevant reports (for CNN [18], and for GenHMM and GMM-HMM [7]) or documentation for the frameworks in which they were implemented (PyTorch for LSTM, sklearn for SVM. Random combinations of increased and decreased values were explored. While ideally each classifier would be repeatedly evaluated on multiple datasets, time constraints would prevent some combinations from being tested. We thus evaluated the classifiers only on dataset $B$ and let the search run for as long as possible.

The values used in section 3.1 .3 are the ones that gave the best result in the hyperparameter search. As most classifier types have an element of randomness it is possible that other settings would have given better results in repeat trials, but given the data at hand these settings are the best guesses available. It should be noted that the SVM search had to be cut short as the training time escalated, and even the classification was so slow that it would be unsuitable in a practical setting. Classification of a single $5 \mathrm{~s}$ sample exceeds $1 \mathrm{~s}$ on the equipment used (see section 3.3 for specifics).

\subsection{Multi-label Study}

Evaluation of the single-label study was performed before the multi-label study commenced. Given the results in section 4.1 the decision was made to continue 
with the LSTM, GMM-HMM and GenHMM for the multi-label classifier comparison.

The three types of additive noise described in section 3.1 are again used as possible labels for our samples. We also consider four additional degradation types:

Infrasound, another type of additive noise which may be caused by ventilation systems in large buildings such as hospitals. It is distinct from Air-conditioning noise in that it only has energy in frequencies below $20 \mathrm{~Hz}$. We generate Infrasound by creating two sine tones in the 2-18 Hz range, adding pink noise, and passing the result through a lowpass filter with a stop frequency of $20 \mathrm{~Hz}$. This is then added to the speech signal.

Random Muting emulates a faulty microphone cable by randomly cutting out 100-750 ms segments until $20 \%$ of the recording has been silenced.

Hard Clipping occurs when the signal level exceeds the maximum of an amplifier or sound card, and causes loss of information in the signal as values outside the range are replaced with the maximum. Since the shape of the waveform is changed we also see a difference in the harmonic content of the signal. To introduce hard clipping into our recordings we scale the signal $\mathbf{x}=\left(x_{1}, \ldots, x_{T}\right)$ so that $20 \%$ of the samples are $\notin(-1,1)$, and then feed it through the transfer function

$$
x_{t}^{\prime}=\min \left(1, \max \left(-1, x_{t}\right)\right) .
$$

Soft Clipping can be caused when an amplifier has a nonlinear response to out-of-range values rather than just cutting them off. It may also be introduced by limiters to avoid hard clipping, as it has less severe effects on the harmonic content of the output. Our soft clipping is implemented by scaling $\mathrm{x}$ so that $20 \%$ of the values are $\notin(-1,1)$ and then passing it through the transfer function

$$
x_{t}^{\prime}= \begin{cases}-2 / 3, & x_{t} \leq-1 \\ x_{t}-\frac{x_{t}^{3}}{3}, & -1<x_{t}<1 . \\ 2 / 3, & x_{t} \geq 1\end{cases}
$$

Compared to Hard Clipping we still lose information in $20 \%$ of the signal but better retain the waveform shape. 
Using these definitions, applying both Hard Clipping and Soft Clipping after one another would result in the same waveform as only applying Soft Clipping. We thus consider these two labels to be mutually exclusive, while the others may be applied independently of one another.

While most hardware used in clinical settings have adjustable recording levels, cheaper configurations may lack such a feature [2]. Accidentally setting the recording level too high, or being restricted to using lower quality hardware, may thus result in some kind of clipping degradation. Similarly, if one has to rely on cheaper or older cables there would likely be a greater risk of corruptions like Random Muting.

\subsubsection{Adapted Dataset Generation}

The dataset generation follows the same general steps as described in section 3.1.1. with TIMIT recordings being corrupted by the ADT. In the multilabel study the prevalence of each corruption can be defined separately, and the corresponding amount of utterances will be given that label. After the Hard/Soft Clipping collisions have been resolved the chosen degradations are applied in a predetermined order:

1. We begin by padding the utterance with $1 \mathrm{~s}$ of silence in the beginning and end, to simulate the silent room before the patient is told to start speaking and before the recording is stopped.

2. Additive noises are added (White Noise, Air-conditioning, Electric Hum, and Infrasound). We use a $40 \mathrm{~dB}$ SNR for all noises except Infrasound, for which we use $20 \mathrm{~dB}$.

3. Random Muting is applied. This ensures that added background noise is also silenced when the hypothetical microphone cable disconnects. It could be argued that Electric Hum may also be introduced at a later stage in the recording setup, but this case has not been considered.

4. Soft or Hard Clipping is applied. This is done after the muting to avoid muting the clipped samples.

5. We scale the resulting signal so that the peak value $\max _{t}\left|x_{t}\right|=a$, for some randomly chosen $a \in[0.5,0.999]$. This emulates a variation in recording volume and makes Soft Clipping less trivial to detect.

The order is chosen to simulate a faulty recording setup as realistically as possible. For instance, it would be unnatural to perform the padding after 
the background noise is added as it is assumed to be independent of the speech. Note that, depending on the prevalence of each label, it is unlikely that all corruptions are applied to any specific sample. The padding (step 1) and scaling (step 5) are always applied, however, even to utterances with no positive labels.

For the multi-label study we considered two dataset versions: one where each corruption had a $12.5 \%$ prevalence, dubbed Realistic, and one where they had a 50\% prevalence, dubbed Balanced. The former is appropriate for evaluation because the label cardinality (average number of labels per sample) is 0.856 , so we have a reasonable amount of uncorrupted recordings while still getting some with multiple corruptions. The label cardinality is lower than the expected 0.875 because only one of Hard and Soft Clipping is kept. The latter, Balanced, has the statistical property of equal prior for positive and negative samples of any one label. At 3.26 its label cardinality is much higher and thus most samples are considerably degraded. We generate two realizations of both Realistic and Balanced for a total of four datasets.

\subsubsection{Classification}

For the multi-label classification we take a different approach to classification. We now associate each corruption with its own binary classifier resulting in a set of seven separate classifiers, each making its own independent decision. For the GMM-HMM and GenHMM this means that fourteen separate models are trained, one for positive and one for negative samples of each label. Note that this means there is no model for recordings without any degradations (the None class in the single-label study) and that both the positive and negative sets for e.g. Electric Hum contain samples with and without Random Muting.

Another approach could have been to consider a single multi-class classifier with $2^{n_{\text {labels }}}$ classes. However, with seven binary labels this results in 128 different classes, which for the HMM-based classifiers also means 128 separate models to train. Given that this does not scale well, and would require much more resources to train and an overhaul of the dataset generation to provide enough training data, we instead opt for the binary classifiers approach. Another benefit to using multiple binary classifiers is the additional flexibility introduced, allowing us to adapt to each unique corruption. For example, it may be easier to detect clipping in the time domain rather than using MFCC features, and for the electric hum a notch filter could help the accuracy by focusing on the most relevant frequencies.

We make some additional changes to the implementations for the multi- 
label study. The LSTM has a slightly reduced learning rate which is compensated for by an increased number of training iterations. We also modify the GMM-HMM implementation to directly derive from the hmmlearn module rather than depend on the work of Liu et al.

\section{Voice Activity Detection}

For the additive noise classifiers the corruption is present throughout the recording, and as they are added at an SNR which is computed as a whole for the entire utterance it may be beneficial to look at only the parts where the spoken signal is relatively weak. To do this we employ a Voice Activity Detector (VAD) and use only the segments flagged as unvoiced during training and testing. An exception is made for Infrasound, as the voice's frequency range rarely extends below $100 \mathrm{~Hz}$ during speech [40]. The use of a VAD is motivated by the assumption that in a realistic use case any disturbances would be so small that the VAD is not severely affected. We noted, however, that for some samples with a high number of corruptions (i.e. multiple positive labels) the VAD would return no unvoiced segments, possibly in part due to the smoothing used and the severity of the clipping degradations.

The VAD we apply is part of WebRTC and is accessed through a Python interface [41]. It operates on $20 \mathrm{~ms}$ frames and uses a running average of 5 frames to determine whether the frame is voiced or not. Extracted segments must be at least $100 \mathrm{~ms}$ long to be considered valid and are discarded otherwise. A dedicated test of the $\mathrm{VAD}$ s $\mathrm{s}$ effect on classification accuracy was performed for the LSTM and GMM-HMM.

\section{Ensemble Classification}

Using an ensemble of classifiers can be a way to increase the classification accuracy. Qi et al. [26] test both model averaging and voting and note that voting slightly improves their results, at the cost of increased training time since additional models need to be trained. As part of the test bed we include a way to train multiple classifiers per corruption and let this ensemble vote on the final label during testing. We experiment with both the LSTM and the GMM-HMM using the same features as in the other tests, but in this case we do not use a VAD. The classifiers in the ensemble train on bootstrapped samples from the given dataset, which means that from a population of $N$ recordings we draw $N$ samples with replacement. 


\subsubsection{Tailored Features}

The inclusion of Infrasound as a possible corruption makes it sub-optimal to use regular MFCCs for all corruption types without taking into account their properties. We thus adapt the MFCCs fed to the Infrasound classifier, whichever algorithm is used, to use the $2-100 \mathrm{~Hz}$ frequency range with 3 bins and 2 cepstra, instead of the regular $20-8000 \mathrm{~Hz}, 23$ bins and 13 cepstra.

Similarly we reduce the number of cepstra used for Random Muting classification to 3, which showed promising results during preliminary testing on the LSTM. This is used in the comparison of the three classification algorithms. We follow up on this with a test comparing regular MFCCs to MFCCs with reduced cepstra (abbreviated as MFCC-3), MFCC-3 with added time difference (or $\Delta$ ), and Root Mean Square (RMS) energy with added time difference. The performance is evaluated on LSTM and GMM-HMM, on both datasets. Both the reduction of the number of cepstra and the inclusion of RMS energy is motivated by Random Muting's effect of silencing the recording, which should be detectable using considerably more simple features than regular MFCCs. We add time difference features as the silencing should be more abrupt than natural sounds, at least in cases where it occurs mid-vowel.

While clipping effects are noticeable in the frequency domain, they affect the time-domain signal more directly. Thus these effects may be easier to detect using other features than MFCCs. An example of such a feature is a signal histogram: given a signal $\mathbf{x}=\left(x_{1}, \ldots, x_{T}\right) \in[-1,1]^{T}$ we split the range $[\min \mathbf{x}, \max \mathbf{x}]$ into 19 evenly spaced bins. We then compute the ratio of samples which belong to each bin, resulting in a feature vector of length 19. This can be done either for frames as with the MFCCs or for the entire signal at once, though the latter case only results in a single feature vector and is less suited for use with HMM or LSTM approaches. We compare the results of framed histograms to MFCCs for both LSTM and GMM-HMM, and to a GMM classifier with full-recording histograms. The approaches are evaluated for both Soft and Hard Clipping.

Finally, we revisit Infrasound using the GMM classifier and MFCC features. In this instance we lower the bound to $2 \mathrm{~Hz}$ but otherwise use the default parameters ( 23 bins and 13 cepstra), and average the values over the entire recording. 


\subsection{Experiments and Evaluation}

PyTorch has built-in support for Nvidia CUDA, making it possible to train the GenHMM, LSTM and CNN on a GPU. We use a GeForce GTX 970 with 4 GB VRAM. The amount of VRAM available was a limiting factor, especially for the GenHMM and in configurations with more parameters during the hyperparameter search, making a reduced batch size necessary for certain tests. The GMM-HMM and SVM were instead trained on an Intel i5-4690K 3.5 GHz CPU, and for some choices of regularization parameter $C$ we noted extremely long training and testing times for the SVM. The hmmlearn GMM-HMM implementation had some issues with divergence during training but the underflow causing the divergence could be identified and corrected.

To evaluate the optimal performance of each classifier in the single-label study we evaluate every instance on the testing set after each trained epoch and compute the mean accuracy. If the mean accuracy has increased since the last epoch we train the classifiers for yet another epoch. If not, training is stopped and the penultimate epoch is used as the basis for comparison. In addition to mean accuracy we also evaluate the interquartile range of the accuracy to determine if the classifiers perform consistently. Ten instances of each type are used per dataset except SVM, which is not trained in multiple epochs but rather fit once, as our SVM had no element of randomness.

In the multi-label study we increase training times and train for a single epoch. As the prior distribution for positive and negative labels are not equal in the Realistic dataset we use the balanced accuracy for evaluation [42]:

$$
\text { balanced accuracy }=\frac{1}{2} \cdot\left(\frac{\text { true positives }}{\text { positives in test }}+\frac{\text { true negatives }}{\text { negatives in test }}\right) .
$$

Since there are two classes for each label, a balanced accuracy of 50\% corresponds to random guessing given the prior distribution. There are other metrics that can be relevant as well, such as the overall precision and recall or the predicted label cardinality.

\subsubsection{Confusion and Specificity Experiment}

In a final experiment the best performing feature and classifier combinations for each individual degradation are chosen and again applied to the Realistic dataset. The goal of this test is to check if the classifiers disproportionately confuse similar corruptions, and whether recordings without any corruptions are reliably labeled as negative by all classifiers. From the results of sec- 
tion 4.2.1, the LSTM was chosen for all corruptions except Soft and Hard Clipping. These instead have a GMM classifier with histogram features (see figures 4.5 and 4.6). The respective reduced cepstra MFCC variants are used as the features for Random Muting and Infrasound. Ensemble classification and VAD filtering are not used. Results are presented in section 4.2.4. 


\section{Chapter 4}

\section{Results}

In this chapter, results are presented and analyzed.

\subsection{Single-label Comparison Study}

Figure 4.1 displays the results of the single-label classifier comparison. Both the LSTM and GMM-HMM perform well in this test, with relatively high average accuracies across all datasets. The $\overline{C N N}$ is less consistent and we noted a large variation in accuracy across repeat trials on any one dataset. Because of this it was not considered in the multi-label case. The performance of the SVM is comparable to the LSTM and GMM-HMM but as stated before both training and testing times were extremely long due to the large number of training vectors, making it impractical for further study. The GenHMM in general has a considerably lower accuracy than the other classifiers, which may be due to the characteristics of the training data or optimal hyperparameter settings not being found during the search. It was used in the multi-label classifier comparison as well to see if it would see an improvement in performance in the new circumstances. 


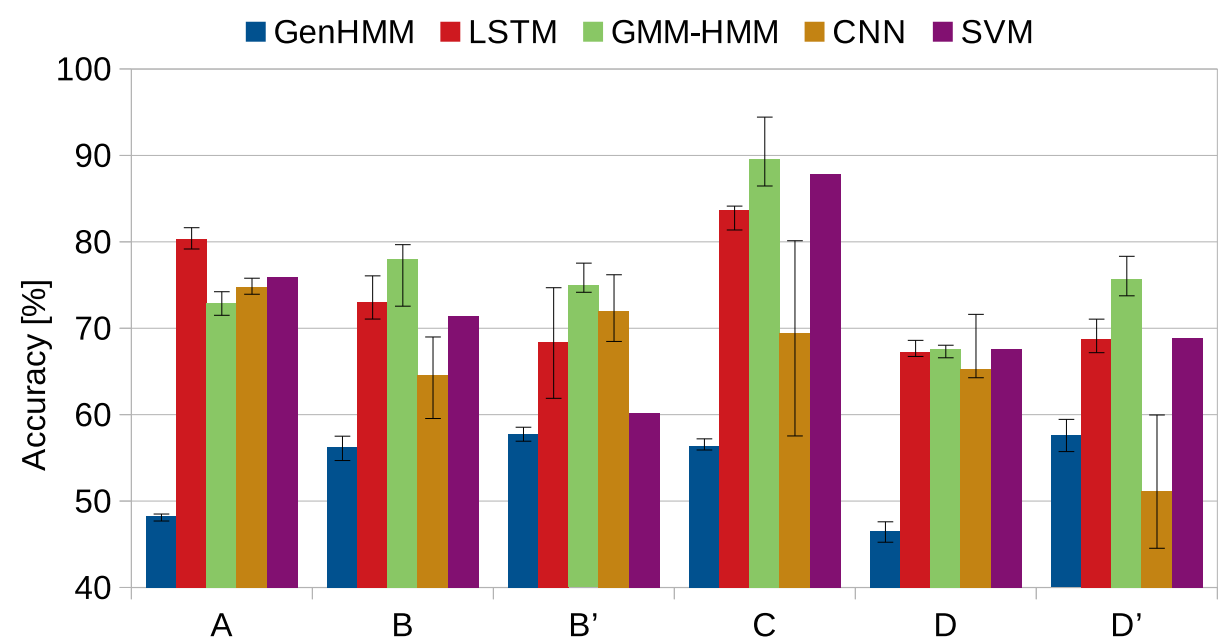

Figure 4.1: Classification accuracies for the five algorithms in the single-label study. Values are computed as the mean of ten initializations, except for the SVM and the error lines show the interquartile range. Note that $C$ has four classes while the other datasets have three.

\subsection{Multi-label Comparison Study}

\subsubsection{Optimal Feature and Classifier}

The results of the multi-label test are shown in figures 4.2 and 4.3 . We note that the LSTM again performs well, especially on Infrasound, while the GMM-HMM has more varied results overall. The GenHMM performs only marginally better than random guessing, though given the results of the singlelabel study it is unsurprising that the GenHMM is outperformed by the other classifiers. The relative success of the LSTM was unexpected, however, as the GMM-HMM had an higher overall accuracy on five out of six datasets in the single-label case. Interestingly, in the multi-label case the LSTM also has a higher balanced accuracy on all three of the corruptions used in the singlelabel test. 


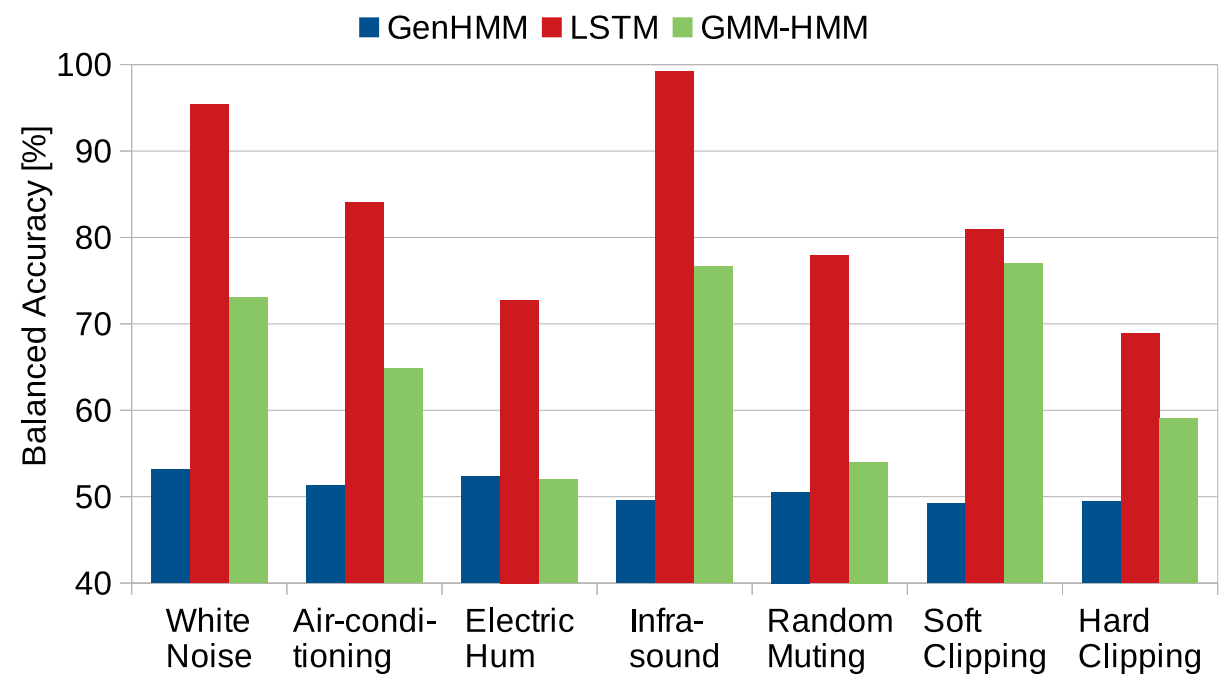

Figure 4.2: The average balanced accuracy across all trials and both realizations on the Realistic dataset.

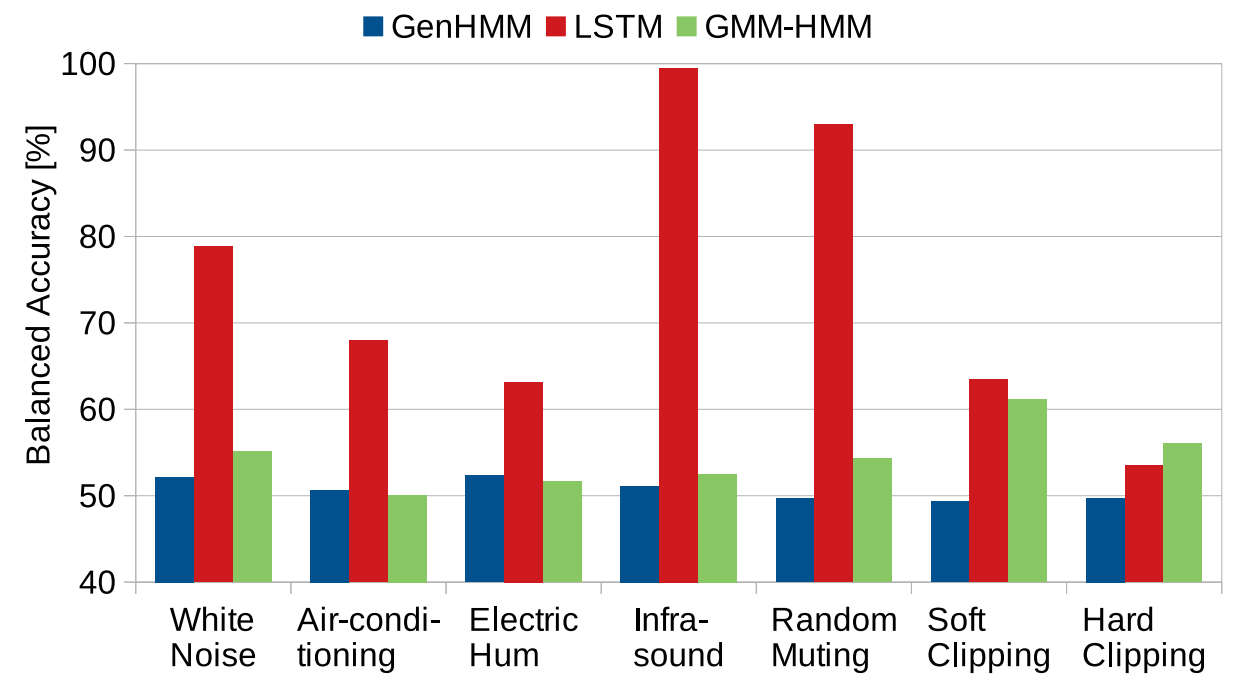

Figure 4.3: The average balanced accuracy across all trials and both realizations on the Balanced dataset. 
In the feature comparison for Random Muting, seen in figure 4.4, we note that the reduced cepstra variants, MFCC- 3 and MFCC $3+\Delta$, offer an improvement over regular MFCCs for the LSTM. While the same is true for MFCC- $3+\Delta$ for the GMM-HMM the variant without the time difference performs equally or worse. We note that the RMS energy is outperformed by all other feature types in all cases.

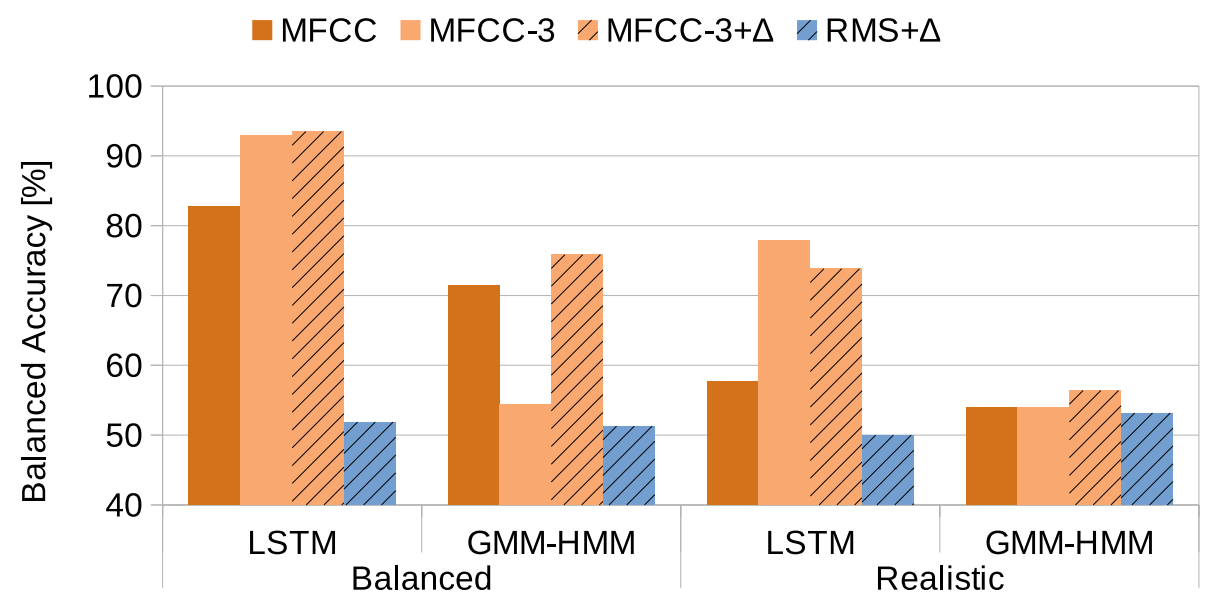

Figure 4.4: Performance of different features in Random Muting classifiers. Striped columns have time difference features appended. MFCC. 3 is a variant with fewer cepstral coefficients described in section 3.2.3.

The results of the histogram feature test for Soft and Hard Clipping are displayed in figures 4.5 and 4.6, respectively. Histogram features consistently improve performance for both LSTM and GMM-HMM on Realistic, but rather the opposite on Balanced. The GMM-histogram combination, however, outperforms all other variants in all tested cases. 


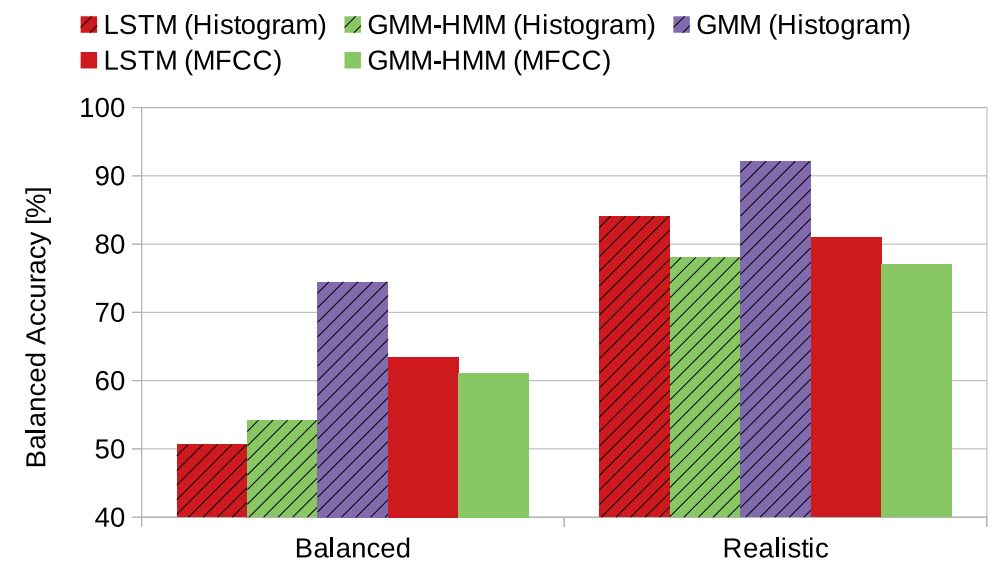

Figure 4.5: Soft Clipping comparison of histogram versus MFCC features on several classifier types. Note that the GMM implementation used histograms of the entire signal, while the LSTM and GMM-HMM used frame-wise histograms.

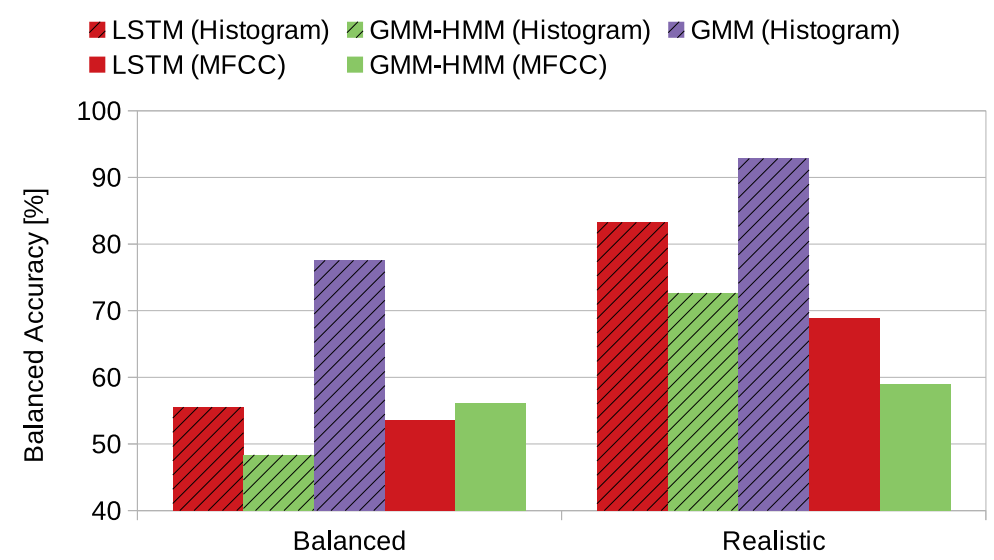

Figure 4.6: Hard Clipping comparison of histogram versus MFCC features on several classifier types. Again, the GMM implementation used histograms of the entire signal, while the LSTM and GMM-HMM used frame-wise histograms. 
$38 \mid$ Results

Finally, in the Infrasound test we note that the GMM approach clearly outperforms the GMM-HMM but does not rival the LSTM. Similarly to the GMM-HMM it performs better on Realistic than on Balanced. These results are shown in figure 4.7 .

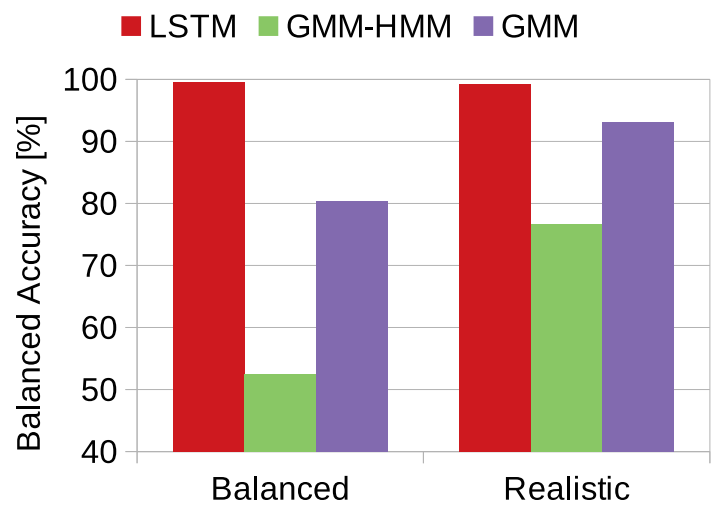

Figure 4.7: Comparison of the 2-8000 Hz MFCCs with a GMM to the 2-100 Hz MFCCS used with the LSTM and GMM-HMM 


\subsubsection{Voice Activity Detection}

The results of the VAD experiment is shown in figure 4.8. We note that in general the LSTM performs worse when the VAD is applied while the GMM$\mathrm{HMM}$ on the other hand benefits from it. Both effects are largest for White Noise on the Balanced dataset.

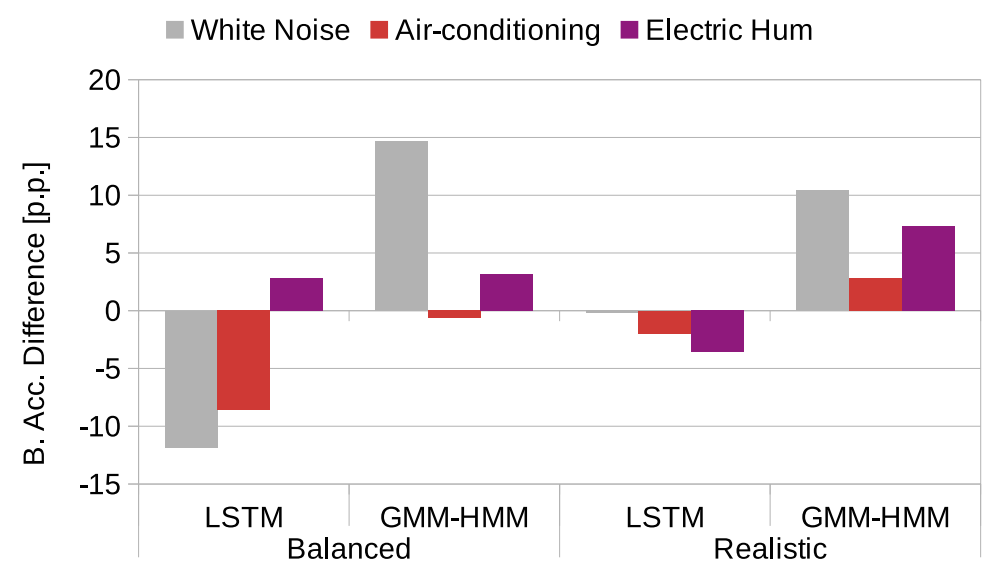

Figure 4.8: The difference in balanced accuracy, measured in percentage points, when VAD filtering (see section 3.2.2) is applied compared to when it is not.

\subsubsection{Ensemble Classification}

The voting model substantially increases the accuracy of the GMM-HMM classifier on certain corruptions. On the Realistic dataset (shown in figure 4.9) we see a noticeable increase for Air-conditioning, Electric Hum, and Infrasound. Accuracy on Hard Clipping is also improved by using ensembles of size 3, but curiously larger ensembles yield worse results. On the Balanced dataset (figure 4.10), the Infrasound and Random Muting corruptions benefit from the voting approach, while Soft Clipping shows the opposite. 
$40 \mid$ Results

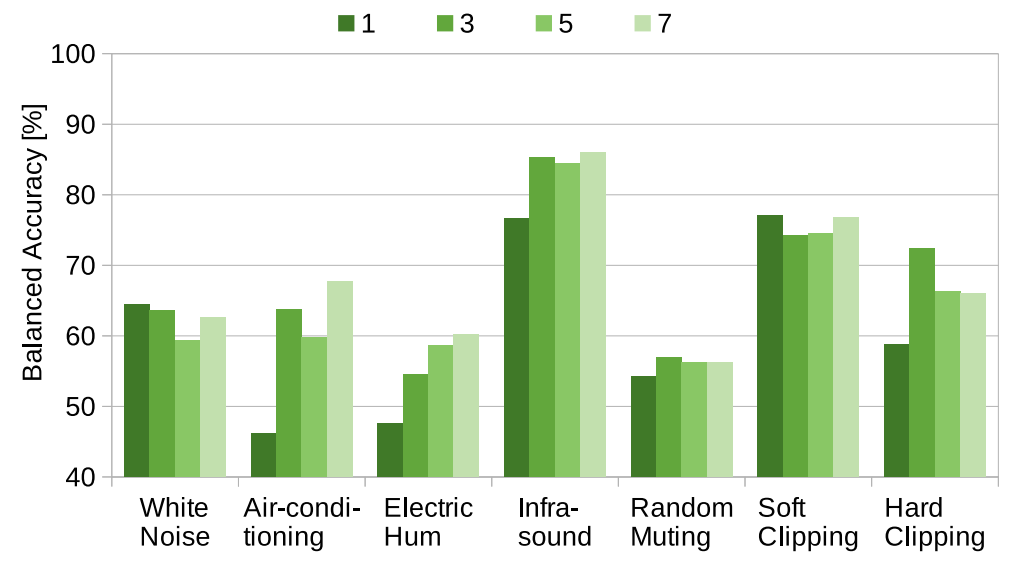

Figure 4.9: Balanced accuracy for each corruption as a function of ensemble size when using voting for the GMM-HMM on the Realistic dataset.

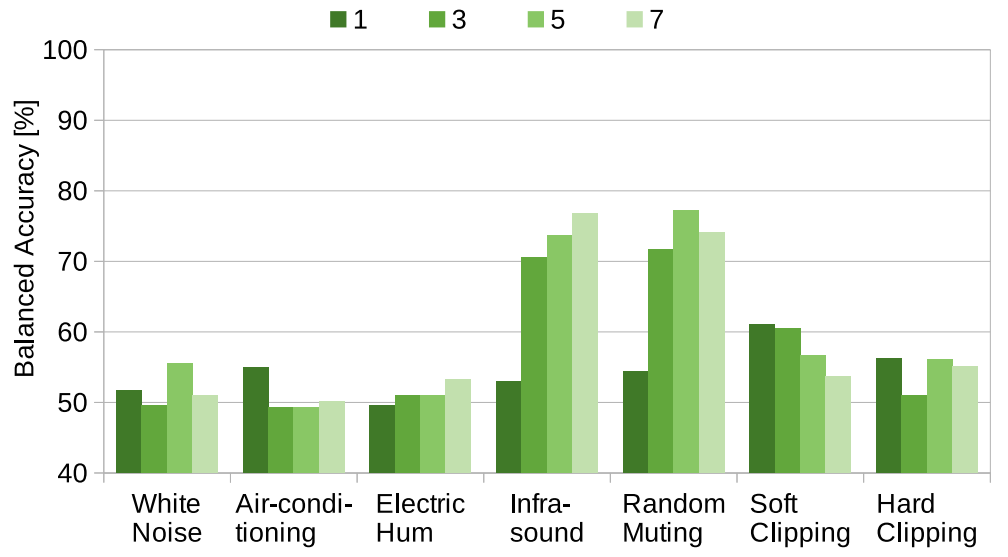

Figure 4.10: Balanced accuracy for each corruption as a function of ensemble size when using voting for the GMM-HMM on the Balanced dataset. 
The LSTM is much less affected by the voting. On the Realistic dataset (figure 4.11) it boosts the accuracy on Random Muting and Soft Clipping, while it is detrimental to classification of Air-conditioning, Electric Hum, and Hard Clipping. The effects on the Balanced dataset (figure 4.12) are marginal across the board.

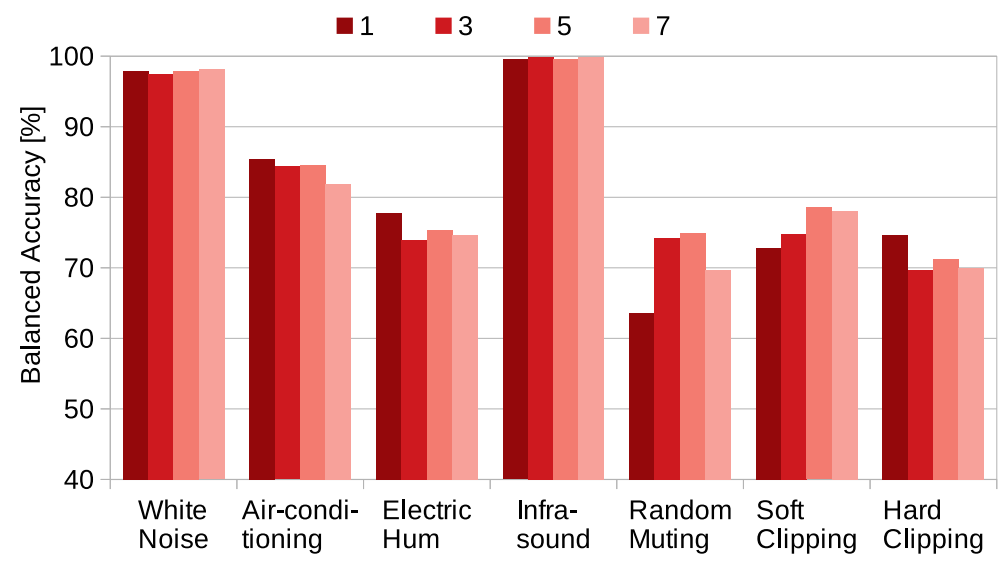

Figure 4.11: Balanced accuracy for each corruption as a function of ensemble size when using voting for the LSTM on the Realistic dataset.

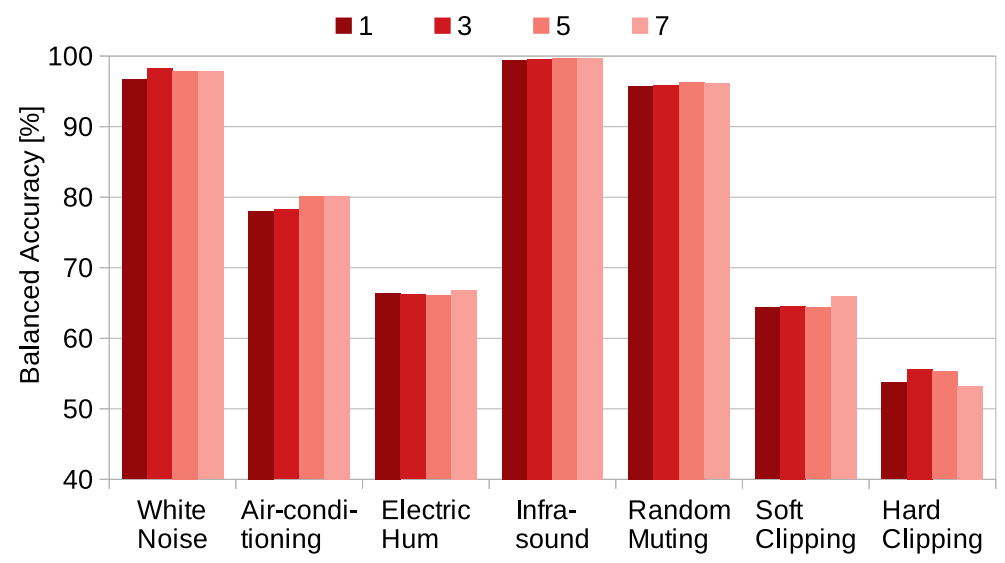

Figure 4.12: Balanced accuracy for each corruption as a function of ensemble size when using voting for the LSTM on the Balanced dataset. 


\subsubsection{Confusion and Specificity Experiment}

The results of the classification of clean recordings are presented in table 4.1. Note that we make no distinction of what corruption was present or detected in this experiment. This means that e.g. a sample with only White Noise that is labeled by the classifier array as only having Soft Clipping would appear in the Any corruption/Any corruption field. The balanced accuracy for detecting clean recordings becomes $93.45 \%$.

Table 4.1: Confusion matrix for clean recordings on the Realistic dataset.

\begin{tabular}{l|rr} 
Predicted & \multicolumn{1}{|l}{ Clean } & Any corruption \\
\hline Clean & $96.65 \%$ & $9.75 \%$ \\
Any corruption & $3.35 \%$ & $90.25 \%$ \\
\hline Total recordings & 3675 & 5775
\end{tabular}

The inter-label confusions are presented in figure 4.13. Note that the numbers displayed along the diagonal represent the true positive rate and not the balanced accuracy used in most earlier figures. The Random Muting classifier, for instance, has a higher true negative rate than true positive rate which is not reflected in this figure. The same is true for the Air-conditioning and Electric Hum classifiers, while the opposite applies to both clipping classifiers.

The baseline is that two labels occur together in about $12 \%$ of the samples, with some variation due to the randomness in label assignment during dataset generation. We can see that Hard and Soft Clipping, which are mutually exclusive, are detected by the other classifier more often than not. Thus, while the GMM-histogram combination is good at detecting whether there is any clipping present, it is worse at determining what type of clipping is present.

Another point of interest is that the White Noise classifier is sensitive to Electric Hum (and Soft Clipping), while the reverse effect is not as strong. There is also significant correlation between the Air-conditioning and Electric Hum labels, which are both based on recordings of noise. 
Results $\mid 43$

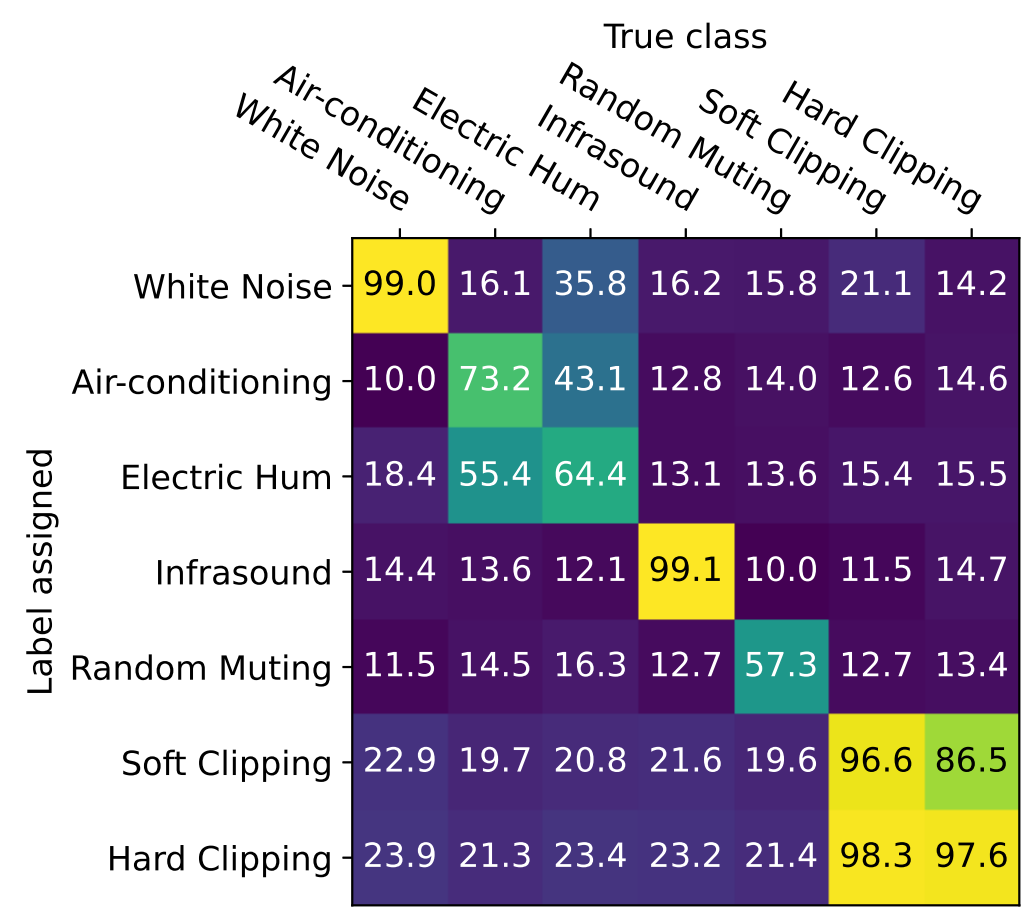

Figure 4.13: Confusion between corruption types on the Realistic dataset. A cell contains the percentage of samples having the column label that are given the row label. Note that labels naturally occur together in about $9 \%$ to $14 \%$ of samples. 


\subsection{Reliability and Validity}

To answer the first research question based on these results we need to make several assumptions. The first is that the hyperparameter search found optimal or close to optimal settings for each classifier. While there were settings left to test when the search was halted, the search was performed randomly so that not only a single part of the setting space would be explored. As we based the boundaries of the search on values found in related reports or documentation we hopefully found decent settings for all classifiers but this is not guaranteed. Another assumption is that the settings used in single-label classification also are appropriate in a multi-label setting. The GMM-HMM $\mathrm{s}$ s poor performance on Electric Hum in the multi-label study could indicate this is not the case, but it could also be that the GMM-HMM is less robust to the influence of the other corruptions.

The validity of the results depend not only on the design choices made in this study, but also on the underlying implementations in PyTorch, hmmlearn, and sklearn and by Liu et al. As previously stated the hmmlearn GMM-HMM had issues with underflow during training and one such issue could be found and corrected. During the VAD comparison another problem surfaced in that there were no checks to detect invalid entries in e.g. the weight matrices, so the code was modified to abort the training and return the most current valid model when such entries are encountered. Considering these two problems were discovered there may be others which were not, as other problems may have only affected the end result rather than thrown exceptions.

An issue was also found in the computation of the generator transform in the GenHMM implementation. During training, when the input vector is split into two parts (eq. 2.2. sec. 2.1.3) the method does not check that the input length is even, instead silently dropping the last dimension if the length is odd. As we used MFCCs with 13 cepstra (following the original report [7]), both in the single-label study and for most corruptions in the multi-label study, some information is neglected by the GenHMM and its results may have been better if the input had been padded. However, this was discovered too late into the project for it to be feasible to re-run the GenHMM measurements. 


\section{Chapter 5}

\section{Discussion and Conclusions}

In this chapter, conclusions are drawn from the findings in the previous chapter, the limitations of the methods chosen are discussed, and possible continuations of this work are presented.

\subsection{Discussion}

The underlying assumption in dataset generation is that the recordings in TIMIT are all devoid of the issues we are trying to introduce. Verifying the entirety of TIMIT is out of scope for this project but no reports of recording issues with TIMIT were found. One concern regarding our datasets, however, is that the amount of data for Air-conditioning and Electric Hum is low: only 15 air-conditioning recordings and 19 electric hum recordings were used. One possible solution would be to generate these sounds as well, but this approach raises new concerns about how to create realistic air-conditioning noise which is varied enough that it is useful for training. A simple way of increasing the variance in the data would be to create pitch-adjusted duplicates of the recordings. One would then need to assure that the pitch adjustment does not cause any other artefacts which could affect the classification.

Overall, the LSTM performs well in the single-label study and outperforms the other algorithms in most multi-label experiments. A notable exception is with the histogram feature for detection of hard and soft clipping, where a GMM provides a higher balanced accuracy. As shown in figure 4.2, using an LSTM in conjunction with MFCC features provides a considerably higher accuracy than chance on corruptions with different characteristics. This suggests that it would be a useful initial combination when detecting a corruption where a specialized feature or algorithm is not immediately 
available.

The LSTM and GMM were used in the experiment detailed in section 3.3.1, and the results thereof are presented in section 4.2.4. Two important conclusions can be drawn: this setup is unlikely to falsely label a clean recording as corrupted, and confusions between different labels are mainly between labels representing similar corruptions. That is, the audible additive noises form one cluster, the clipping corruptions form another, and Infrasound and Random Muting are largely unaffected by the others. Notably these clusters also use the same features.

This is a good result regarding the practical usage of the tool. In practice the exact kind of additive noise or characteristic of the clipping may not matter as the rectifying course of action would still be to insulate the room or reduce the gain, respectively. A significant overlap between e.g. Random Muting and Soft Clipping would have been more problematic. Indeed, a possible iteration of this tool would be to have a single binary classifier for additive noise and then, if needed, classify the detected background noise. The same could apply to the clipping corruptions.

Compared to the other algorithms the GenHMM performs poorly in the tests, and in the multi-label study it hovers around a balanced accuracy of $50 \%$. This is equivalent to random guessing given the a priori distribution for the samples, suggesting that it fails to learn the distinguishing marks of the corruption in question. Possible reasons include that it had too many parameters to train or was not allowed to train for long enough. However, an attempt at reducing both the hidden dimension of the networks and chain length while increasing training time showed no significant improvement. The generator transform computation issue described in section 4.3 may also have affected the results negatively.

As mentioned in section 4.3, no separate hyperparameter search was conducted for the multi-label study. It is possible that the results of both the GenHMM and GMM-HMM are negatively affected by this in a way that the LSTM is not. The increased complexity of the samples could need a higher number of states to accurately model, as, for example, an Electric Hum classifier must be invariant to whether Soft Clipping is present. Further, the corruptions are possibly ill-suited for left-to-right HMMs (as used with the GenHMM as none of them are sequential in nature: the additive noises are continuous, muting can occur whenever, and so on. This differs from the GenHMM s original use case of phoneme recognition. 


\section{Voice Activity Detection}

Judging by the results in section 4.2.2 our implementation of VAD filtering does not cooperate well overall with the LSTM. Recall that we split each file into segments of unvoiced audio, classifying each file separately and finally averaging the result. This filtering possibly disrupts the LSTM algorithm as its hidden state and memory cell is being reset for each segment, removing the possibility of capturing long term contexts between the segments. For the GMM-HMM on the other hand, successful filtering may reduce the complexity of the data being modeled and possibly counteract the need for more states as mentioned above. An option that could improve the filtering results for the LSTM would be to concatenate the unvoiced segments and again treat them as a single recording. This would however introduce new transients where the segments are joined.

\subsection{Sustainability and Ethics}

The method for analyzing speech signals presented in this study could have a number of potential benefits to society. First of all it could help make vocal treatment and research participation more equal to all by improving the quality of service in smaller or more remote clinics. Even if the recording setup or environment is such that a disturbance cannot be removed outright, such as the intrinsic noise in lower-grade equipment, the tool could help analyze the signal so that the detected problems can be taken into account and compensated for. An extension of this would be to adapt the tool for telemedicine purposes, so that it can guide patients who need to make a recording on their own using a smartphone or computer headset. This could help in cases where a clinic is too remote or the patients are otherwise unable to travel.

From a sustainability perspective this study would mainly contribute to target 3.8 of the United Nations' Sustainable Development Goals, given the above reasoning. This target reads: "achieve universal health coverage, including financial risk protection, access to quality essential health-care services and access to safe, effective, quality and affordable essential medicines and vaccines for all" (emphasis added) [43]. By contributing to making treatment for vocal disabilities more accessible it may also benefit target 10.2, which reads "by 2030, empower and promote the social, economic and political inclusion of all, irrespective of age, sex, disability, race, ethnicity, origin, religion or economic or other status" [43].

Since we have used an established corpus in this study we have avoided 
the integrity problems that come with using real medical data. Even though this tool operates without any need for information about the subject's identity or potential disorder, the intended use case is in a clinical setting and voice recordings of the kind we use are hard to anonymize. Additionally, if one were to use algorithms that require a lot of computational resources during classification the tool would likely need to run on a special server, rather than existing lab computers in clinics. In this case one would also need to consider how to transfer the recordings and store them securely on the server. However, the computationally heavy part is mainly the classifier training. Once the training is complete the algorithms used in this study would have no problem classifying a handful of samples if implemented in a regular web server or even in a smartphone application.

We should also note that the training data itself is not needed for classification. Thus the data can be destroyed after the algorithms have been trained, or the training can be done in a trusted environment where the data is kept and only the trained classifiers sent out to clinics. Similarly, if we use certain features (such as the histogram or reduced-cepstra MFCCs) we are likely not able to reproduce the signal from the features in a way that allows the speaker to be identified. If we are performing classification on a web server we could then extract the features client-side and consequently not have to transmit any identifiable data.

\subsection{Limitations}

While the dataset generation tool and test bed could be considered usable, practical usage in clinics is limited at the moment. Firstly, all recordings in TIMIT are short (less than $8 \mathrm{~s}$ ) spoken phrases designed to be phonetically diverse, and they are all spoken in various dialects of American English. A speech therapist may encounter problems with this tool when working in another language or using some other exercise than simply speaking a phrase, as those types of recordings do not occur in the training data. One could use a spoken calibration phrase in English to test the equipment before a patient evaluation takes place, but that would not be possible if one wants to verify the quality of older recordings which do not adhere to this format.

Secondly, speech therapists would in normal cases see patients with some sort of condition, which is why the recordings are being made in the first place. While TIMIT does feature some speakers with colds or "hoarse" voices and one or two with voice disorders, it is possible that certain disorders confuse the classifiers as they are not present in the training data. It is reasonable to 
assume, however, that there would be little increase in false negatives as voice disorders are unlikely to counteract the issues which are being tested for.

Regarding the design of the corruptions there are certain choices which may have been done differently. At $20 \%$ of samples clipping, both clipping corruptions are rather severe and would easily be detected by a human listener, especially compared to Infrasound. It would be interesting to see the correlation between the number of samples clipping and the classification accuracy, and whether the GMM still outperforms the LSTM at lower percentages. The same reasoning applies to Random Muting.

The assumption made when using voice activity detection is that the VAD is likely developed to be robust against small disturbances. In a realistic use case, where corruptions are subtle enough to not be easily detectable by ear and an external tool is needed, the VAD would still have good accuracy. In our case, especially with the Balanced dataset and the clipping degradations, this is possibly not the case and the VAD test results may be negatively affected by the voiced/unvoiced classification accuracy of the VAD itself. As the entire TIMIT corpus is annotated one could design a VAD accuracy test, comparing the corrupted and original recordings.

\subsection{Future work}

There are multiple ways of improving or continuing upon the work in this study. Adding new corruptions or testing other feature types or classification algorithms is relatively easy with the current framework, and the procedure for doing so is described in the appendix. As mentioned above, one continuation would be to arrange the classification in a hierarchical manner by first using general classifiers of groups of corruptions such as additive noise or clipping. One would then use another round of classification for the samples that test positive to determine the exact type of additive noise.

An interesting alternative for the HMM classifiers would be to instead train one model each for the corruptions and one for clean speech, and during classification yield all labels whose model scores higher than the clean model. This is more similar to the classification method used in the single-label case which might benefit the GMM-HMM. It would also have the added bonus of reducing training time as fewer models need to be trained, which was a bottleneck for the GenHMM. Note that in this case one cannot use different features for the different models, but it could work well with the second round of classification in a hierarchical setup.

One drawback of our current test setup is the similarity between the 
training and testing set. It would be interesting to investigate more thoroughly whether the classifiers have actually learned what we intended for them to learn. Would the clipping classifiers, trained as in this study, reliably detect samples with less amounts of clipping or if we reduce the volume by a factor $\frac{1}{10}$ ? Are the classifiers robust to new, unseen types of corruptions such as a high-pass filter? These and other questions are possible continuations of this study. Of course, it would also be interesting to test the classifiers on authentic data and to see if the knowledge from training on generated datasets is valid in the real world as well.

\subsection{Conclusions}

Overall, machine learning approaches are useful for the detection of signalrelated corruptions in voice recordings. On an artificial, though reasonably realistic, dataset we achieve at least a $75 \%$ balanced accuracy on each corruption investigated. Long Short-term Memory performs well compared to the other algorithms, peaking at over $99 \%$ balanced accuracy on infrasound, but a Gaussian Mixture Model is better for detecting clipping. We expect the LSTM to also generalize well to corruptions not investigated in this study. The choice of features is important and while MFCCs are a good starting point for these corruptions, tailoring the feature to the specific corruption can increase the accuracy. Ensemble classification is largely unnecessary for the LSTM but helped the GMM-HMM in certain cases, and the same is true for using a Voice Activity Detector to filter out voiced parts of the signal. 


\section{Bibliography}

[1] S. Ternström, P. Pabon, and M. Södersten, "The voice range profile: its function, applications, pitfalls and potential," Acta Acustica united with Acustica, vol. 102, no. 2, pp. 268-283, 2016, ISSN: 1610-1928.

[2] A. P. Vogel and A. T. Morgan, "Factors affecting the quality of sound recording for speech and voice analysis," International journal of speech language pathology, vol. 11, no. 6, pp. 431-437, 2009, ISSN: 1754-9507.

[3] M. P. Gelfer and D. M. Fendel, "Comparisons of jitter, shimmer, and signal-to-noise ratio from directly digitized versus taped voice samples," Journal of Voice, vol. 9, no. 4, pp. 378-382, 1995, ISSN: 08921997.

[4] C. P. Carson, D. R.-S. Ingrisano, and K. D. Eggleston, "The effect of noise on computer-aided measures of voice: a comparison of CSpeechSP and the multi-dimensional voice program software using the CSL 4300B module and multi-speech for Windows," Journal of Voice, vol. 17, no. 1, pp. 12-20, 2003, ISSN: 0892-1997.

[5] B. Barsties and M. De Bodt, "Assessment of voice quality: current stateof-the-art," Auris Nasus Larynx, vol. 42, no. 3, pp. 183-188, 2015, ISSN: 0385-8146.

[6] Y. Hu and P. C. Loizou, "Subjective comparison and evaluation of speech enhancement algorithms," Speech communication, vol. 49, no. 78, pp. 588-601, 2007, ISSN: 0167-6393.

[7] D. Liu, A. Honoré, S. Chatterjee, and L. K. Rasmussen, "Powering hidden markov model by neural network based generative models," in 24th European Conference on Artificial Intelligence (ECAI), 2020, ISBN: 978-1-64368-100-9. 
[8] C. M. Bishop, Pattern recognition and machine learning, ser. Information science and statistics. New York, NY: Springer, 2006, ISBN: 0387310738 .

[9] C. Couvreur, V. Fontaine, P. Gaunard, and C. G. Mubikangiey, “Automatic classification of environmental noise events by hidden markov models," Applied Acoustics, vol. 54, no. 3, pp. 187-206, 1998, Issn: 0003-682X.

[10] P. Maijala, Z. Shuyang, T. Heittola, and T. Virtanen, "Environmental noise monitoring using source classification in sensors," Applied Acoustics, vol. 129, pp. 258-267, 2018, ISSN: 0003-682X.

[11] C. Kotropoulos and S. Samaras, "Mobile phone identification using recorded speech signals," in 2014 19th International Conference on Digital Signal Processing, IEEE, 2014, pp. 586-591, ISBN: 978-1-47994612-9.

[12] L. Ma, B. Milner, and D. Smith, "Acoustic environment classification," ACM Transactions on Speech and Language Processing (TSLP), vol. 3, no. 2, pp. 1-22, 2006, ISSN: 1550-4875.

[13] L. Dinh, J. Sohl-Dickstein, and S. Bengio, "Density estimation using real NVP," arXiv preprint, 2016. arXiv: 1712.00866.

[14] N. Morgan and H. Bourlard, "Neural networks for statistical recognition of continuous speech," Proceedings of the IEEE, vol. 83, no. 5, pp. 742772, 1995, ISSN: 0018-9219.

[15] Torch Contributors. (2019). "Pytorch documentation," [Online]. Available: https: / / pytorch .org/docs / stable/index.html (visited on 2020-11-30).

[16] S. Hochreiter and J. Schmidhuber, "Long short-term memory," Neural computation, vol. 9, no. 8, pp. 1735-1780, 1997, ISSN: 1530-888X.

[17] M. S. Hussain and M. A. Haque, "SwishNet: a fast convolutional neural network for speech, music and noise classification and segmentation," arXiv preprint, 2018. arXiv: 1812.00149

[18] E. Cakir, G. Parascandolo, T. Heittola, H. Huttunen, and T. Virtanen, "Convolutional recurrent neural networks for polyphonic sound event detection," IEEE/ACM Transactions on Audio, Speech and Language Processing (TASLP), vol. 25, no. 6, pp. 1291-1303, 2017, ISSN: 23299290. 
[19] J. Lee, T. Kim, J. Park, and J. Nam, "Raw waveform-based audio classification using sample-level CNN architectures," arXiv preprint, 2017. arXiv: 1712.00866 .

[20] scikit-learn developers. (2020). "Support vector machines," [Online]. Available:https://scikit-learn.org/stable/modules/ svm.html (visited on 2020-11-18).

[21] S. Davis and P. Mermelstein, "Comparison of parametric representations for monosyllabic word recognition in continuously spoken sentences," eng, IEEE Transactions on Acoustics, Speech, and Signal Processing, vol. 28, no. 4, pp. 357-366, 1980, ISSN: 0096-3518.

[22] L. R. Rabiner and R. W. Schafer, Introduction to digital speech processing. Now Publishers Inc, 2007, ISBN: 9781601980700.

[23] S. Hershey, S. Chaudhuri, D. P. W. Ellis, J. F. Gemmeke, A. Jansen, R. C. Moore, M. Plakal, D. Platt, R. A. Saurous, B. Seybold, M. Slaney, R. J. Weiss, and K. W. Wilson, "CNN architectures for large-scale audio classification," in 2017 IEEE International Conference on Acoustics, Speech and Signal Processing (ICASSP), IEEE, 2017, pp. 131-135, ISBN: 978-1-5090-4117-6.

[24] K. El-Maleh, A. Samouelian, and P. Kabal, "Frame level noise classification in mobile environments," in 1999 IEEE International Conference on Acoustics, Speech, and Signal Processing (ICASSP), vol. 1, IEEE, 1999, pp. 237-240, ISBN: 0780350413.

[25] V. Peltonen, J. Tuomi, A. Klapuri, J. Huopaniemi, and T. Sorsa, "Computational auditory scene recognition," in 2002 IEEE International Conference on Acoustics, Speech, and Signal Processing (ICASSP), vol. 2, IEEE, 2002, pp. 1941-1944, ISBN: 0-7803-7402-9.

[26] S. Qi, Z. Huang, Y. Li, and S. Shi, "Audio recording device identification based on deep learning," in 2016 IEEE International Conference on Signal and Image Processing (ICSIP), 2016, pp. 426-431, ISB N: 9781-5090-2377-6.

[27] A. Leman and J. Faure, Method and device for classifying background noise contained in an audio signal, Patent, Orange SA, 2015. [Online]. Available: https : / / patents . google . com / patent / US 8972255 B2 / en (visited on 2020-04-06). 
[28] C. C. E. d. Abreu, M. A. Q. Duarte, and F. Villarreal, "An immunological approach based on the negative selection algorithm for real noise classification in speech signals," International Journal of Electronics and Communications (AEÜ), vol. 72, pp. 125-133, 2017, ISSN: 14348411.

[29] D. Li, I. K. Sethi, N. Dimitrova, and T. Mcgee, "Classification of general audio data for content-based retrieval," Pattern Recognition Letters, vol. 22, no. 5, pp. 533-544, 2001, ISSN: 0167-8655.

[30] A. Rix, J. Beerends, D.-S. Kim, P. Kroon, and O. Ghitza, "Objective assessment of speech and audio quality - technology and applications," IEEE Transactions on Audio, Speech, and Language Processing, vol. 14, no. 6, pp. 1890-1901, 2006, ISSN: 1558-7916.

[31] Z. Li, J.-C. Wang, J. Cai, Z. Duan, H.-M. Wang, and Y. Wang, "Nonreference audio quality assessment for online live music recordings," in Proceedings of the 21 st ACM international conference on Multimedia, 2013, pp. 63-72, ISBN: 978-1-4503-2404-5.

[32] ITU Telecommunication Standardization Sector (ITU-T), “P.563 : singleended method for objective speech quality assessment in narrow-band telephony applications," Series P: Telephone Transmission Quality, Telephone Installations, Local Line Networks, Tech. Rep., 2004.

[33] A. R. Avila, H. Gamper, C. Reddy, R. Cutler, I. Tashev, and J. Gehrke, "Non-intrusive speech quality assessment using neural networks," in 2019 IEEE International Conference on Acoustics, Speech and Signal Processing (ICASSP), IEEE, 2019, pp. 631-635, ISBN: 978-1-47998131-1.

[34] H. Nylén, Degree project prestudy, GitHub Repository, https : / / github.com/HelmerNylen/prestudy, 2020.

[35] N. Harte, E. Gillen, and A. Hines, "TCD-VoIP, a research database of degraded speech for assessing quality in VoIP applications," in 2015 Seventh International Workshop on Quality of Multimedia Experience (QoMEX), IEEE, 2015, pp. 1-6, ISBN: 1479989584.

[36] M. Mauch, S. Ewert, et al., "The audio degradation toolbox and its application to robustness evaluation," in Proceedings of the 14th International Society for Music Information Retrieval Conference (ISMIR 2013), 2013, pp. 83-88, ISBN: 978-0-615-90065-0.

[37] Freesound team. (2005). "Freesound," [Online]. Available: https : // freesound.org/(visited on 2020-11-27). 
[38] H. Nylén, Detecting signal corruptions in voice recordings for speech therapy, GitHub Repository, https : / / github . com/HelmerNylen / speech-signal-issues, 2020.

[39] D. Povey, A. Ghoshal, G. Boulianne, L. Burget, O. Glembek, et al., "The Kaldi speech recognition toolkit," in IEEE 2011 Workshop on Automatic Speech Recognition and Understanding, IEEE Signal Processing Society, 2011, ISBN: 9781467303668.

[40] H. Traunmüller and A. Eriksson, "The frequency range of the voice fundamental in the speech of male and female adults," Unpublished manuscript, 1995. DiVA: diva2:176809,

[41] J. Wiseman, Py-webrtcvad, GitHub repository, https: / / github. com/wiseman/py-webrtcvad, 2019. Accessed 2020-04-13.

[42] K. H. Brodersen, C. S. Ong, K. E. Stephan, and J. M. Buhmann, "The balanced accuracy and its posterior distribution," in 2010 20th International Conference on Pattern Recognition (ICPR), IEEE, 2010, pp. 3121-3124, ISBN: 1424475422.

[43] UN General Assembly, "Transforming our world: the 2030 agenda for sustainable development," A/RES/70/1, 2015. [Online]. Available: https://sdgs.un.org/2030agenda (visited on 2021-01-28). 
56| Bibliography 


\section{Appendix: Documentation}

This is the documentation for the tool developed for and used in this study. Note that this concerns the multi-label code, available at https://github.com/HelmerNylen/speech-signal-issues, while the single-label code can be found at https://github.com/HelmerNylen/prestudy.

To get started with the tool you do not need to read this entire document as much of the content is here for reference. Installation and the first section of Running Experiments are recommended.

\section{Table of contents}

1. Installation

2. Terminology

\section{Structure}
1. classifier
2. datasets
3. degradation
4. features
5. noise
6. noise_classes
7. timit

\section{Running Experiments}

1. Generating Datasets

2. Adding Corruptions

3. Adding Features

- Voice Activity Detection

4. Adding Classifiers

- Ensemble Classification 


\section{Installation}

Ubuntu 18.04.5 was used during development.

1. Clone or download this repository.

2. Install Python 3.6. Version 3.6.9 was used during development.

3. Install NVidia CUDA.

4. Install LLVM via sudo apt-get install llvm.

5. Install SoX and additional audio format handlers via sudo apt-get install sox libsox-fmtall.

6. Install Matlab.

7. Acquire TIMIT and place the root directory (containing DOC, TEST, TRAIN and README. DOC) in the folder timit.

8. Install Kaldi.

9. Decide if you need the GenHMM:

- If you want to use the GenHMM, clone or download the gm_hmm repository and place its root directory in the folder classifier/gm_hmm. Follow its installation instructions. The dataset preparation steps etc. are not needed but can be useful for verifying the installation.

- If you are not interested in using the GenHMM, proceed to the next step. It can be installed at a later time if you change your mind.

10. Open a terminal in this project's root folder (where README.md is located). Create a virtual environment with a Python 3.6 interpreter via python3.6 -m venv pyenv.

11. Activate the environment via source pyenv/bin/activate.

12. Go to [your Matlab root]/extern/engines/python/and run python setup.py install.

13. Return to this project's root folder and install the dependencies listed in requirement s . $t x t$, via e.g. pip install - $r$ requirements.txt.

14. Download the Audio Degradation Toolbox (ADT). Place the content of audio-degradation-toolbox in degradation/adt. The structure should be that degradation/adt now contains three folders: AudioDegradationToolbox, html_gen, and testdata, as well as a number of files.

15. Run degradation/create_dataset . py prepare to convert noise files (recordings for airconditioning noise and electric hum are included) to the right formats, convert the TIMIT files from NIST sphere to standard wavefile etc.

16. Given that the above steps completed successfully, you should now be all set to run . /experiments.py.

To run experiments in a new terminal window, or after restarting your computer, you need to activate the virtual environment using source pyenv/bin/activate.

\section{Terminology}

The terminology used in the codebase is slightly different from that in the report.

- In general, a corruption (report) is here referred to as a noise type or noise class (code). Degradations in the code are generally the smaller operations which constitute a corruption, but can also be used as a third synonym as there is usually only one degradation per corruption.

- A multiclass model in the code is essentially a discriminative model in the report, as the models which are not multiclass only model a single class in a generative way. 


\section{Structure}

The root folder contains some project-wide settings such as the module requirements and linting options. The highest-level interface, experiments . py, is also located here and helps perform more complex tests. It can generate the required datasets and train multiple classifier instances and average their results. Results are saved to a folder called testresults.

\section{classifier}

classifier contains interfaces to or implementations of the various classifier algorithms. classifier . py is an interface for passing data to algorithms, saving/loading trained algorithms, and performing labeling. Using generative models (GMM-HMM or GenHMM), the classifier trains one model for each possible class (i.e. positive and negative). Using discriminative models such as the LSTM, only one model is trained and the Classifier class is mostly just a wrapper.

Each algorithm inherits from Model, defined in model . py. The algorithms themselves are stored in model_x.py files. Algorithm implementations should override the train ( ) and score ( ) methods. Discriminative models should also override get_noise_types ( ) and set the class property MULTICLASS to True. Note that for discriminative models the constructor also receives a noise_types parameter, which is a list mapping label index to corruption ids. This should be stored and returned verbatim by get_noise_types( ).

Default hyperparameters for the algorithm are stored in defaults . j son. Each algorithm should define the "parameters" and "train" fields. These two fields are passed in a dictionary to the constructor and the train ( ) method of a model via the config parameter. Optionally, the "score" field can be used to pass keyword arguments to the score ( ) method.

confusion_table.py defines a confusion table on which precision and other evaluation metrics can be computed. They are used to pass test results from the Classifier class to higher-level interfaces but can also be printed to the terminal directly.

The models folder is the suggested and default place to store trained classifier algorithms. Classifiers are stored here with the . classifier file extension. The Noise Classes interface (see below) also stores its classifier bundles here, using the . noiseclasses extension.

The gm_hmm folder, if present, should contain a clone of the GenHMM repository.

\section{datasets}

This is the suggested and default folder to store all datasets. Assuming you generate a dataset named MyDataset, a folder datasets/MyDataset will be generated. This will contain the training set under datasets/MyDataset/train and the testing set under datasets/MyDataset/test. These two folders each contain a large number of . wav files as well as labels . csv containing comma-separated labels for each file, and degradations . mat which is a complete list of the arguments sent to the Audio Degradation Toolbox. The latter can be useful for debugging if you have a sample and want to know what TIMIT file it originated from or what value was chosen for a random parameter.

Finally, the dataset definition is copied to datasets/MyDataset/source.json. More on how these work below. 


\section{degradation}

This contains the scripts needed to generate datasets. These are accessed via the create_dataset . py tool, the usage of which is explained under section Generating Datasets.

balanced_dataset.json and basic_dataset. j son contain the definitions of the Balanced and Realistic datasets, respectively, used in the report. These are just examples and can be replaced with your own definition or removed.

create_samples . $m$ is a Matlab script that receives instructions form create_dataset . py, invokes the degradations in the ADT, and saves the resulting samples to disk.

degradations . py contains a list of all valid degradations. It helps convert the JSON definition of a dataset into proper arguments for create_samples.m.

preparations . py is an interface to SoX, which helps convert the audio files (TIMIT and noise files) to valid 16-bit $16 \mathrm{kHz}$ mono wavefiles.

The adt folder contains the Audio Degradation Toolbox, which is a Matlab tool with scripts for common degradations.

The custom-degradations folder contains additional degradations. Custom degradations should follow the naming convention and argument signature of the built-in degradations to be detected by

create_samples.m.

\section{features}

The features folder contains tools to extract, filter, and cache various types of features.

mfcc . py contains hooks to external libraries for MFCC features. cust om . py contains definitions for other types of features.

feature_extraction. py is responsible for loading datasets, resolving caching, and invoking the features defined in $\mathrm{mfcc}$. py and custom. py. The extracted features are generally returned as a tuple ( feats, index). feats is a list of Numpy arrays containing feature values, usually with the time dimension along axis 0 (if applicable). index is a list mapping the arrays in feats to the corresponding file in the dataset. It is usually equal to $\mathrm{np}$. arange ( len (feats ) ), but if VAD filtering is used it may look more like $[\odot, \odot, 1,1,1,2,3,3, \ldots]$.

utils . py contains helper functions for the other files.

vad . py performs the voice activity detection filtering. If used it writes each detected segment to a temporary file, and feature_extraction. py extracts the features for each segment and organizes the index variable.

The cache folder stores computed features so that they can be reused without having to recompute them. The size of the cache can be adjusted in feature_extraction . py.

kaldi contains the Kaldi library, used for MFCC extraction. 
noise

noise is where the audio files for additive noise are stored. The name of each subfolder serves as an identifier, and during dataset generation each subfolder is partitioned into a training and a testing set. This does not, however, affect the contents in the noise subfolders on disk.

Only . wav files are considered valid, so to include for example an MP3 file you need to run degradation/create_dataset. py prepare. This creates a . wav copy of each file which conforms to the requirements (16 bits, $16 \mathrm{kHz}$ etc.), however if the original file is also a . wav file it is overwritten.

noise_classes

This folder contains the main tools for working with classifier and feature combinations, as well as definitions of different corruptions (herein referred to as noise classes).

noise_class . py contains tools for classification using one or more classifiers for a specific corruption. A NoiseClass stores trained classifiers, feature and ensemble classification settings, and the degradations which consitute a corruption.

interface. py is used for working with Noiseclass instances. It can be used to train and test classifiers on specific datasets, perform classification of external files using trained classifiers, and to check whether the noise_classes . j son file, the trained classifiers in classifier/models, and the dataset definition in datasets/MyDataset/source . json are in sync, taking into account classifier/defaults . json.

noise_classes . j son is where most of the editing goes when performing different experiments. It contains an array of corruption specifications, which in turn consist of an identifier, a readable name, the degradations needed to create it, and the classifier(s) and features that should be used to detect it. The identifiers in this file are used in the dataset definitions. See Adding Corruptions for more details.

\section{timit}

The timit folder contains the uncorrupted speech samples used to make the datasets. All sound files encountered in this folder (searching all subfolders recursively) are converted to conforming . wav files during the data preparation step. The separation into test and training set in TIMIT is ignored when new datasets are created. If you want to add other speech files to the datasets you can place them here, but note that the new files should also be free from any corruptions. 


\section{Running Experiments}

If you have followed the installation instructions it should be possible to run the experiments . py script in the root folder. In the beginning of this file there are four constants that you can change to run different experiments.

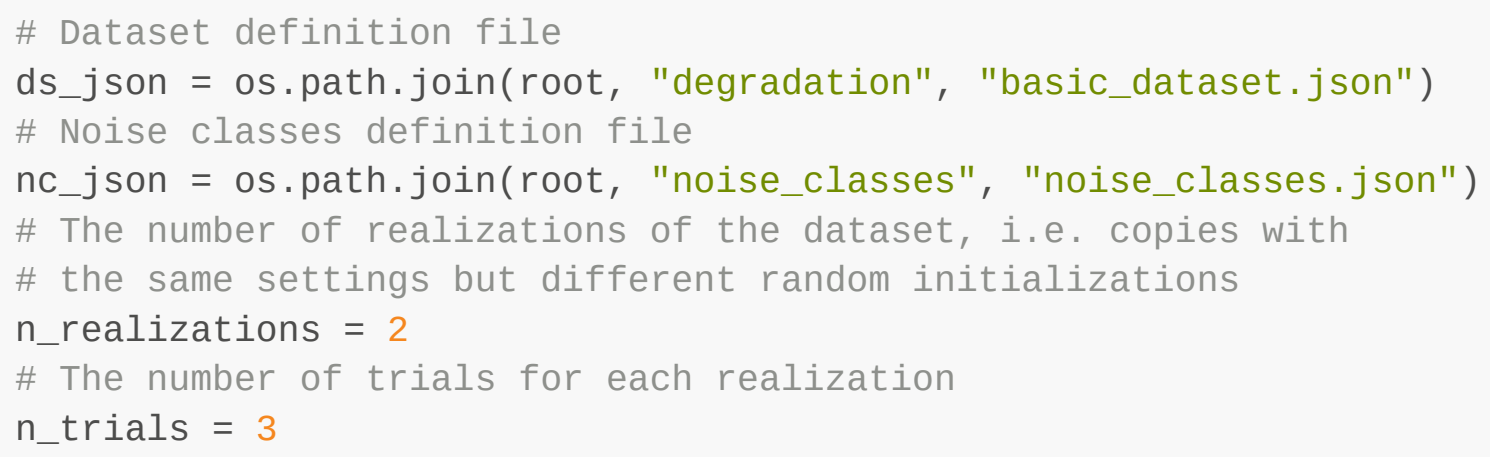

For example, if you want to average the results over more repeated trials you can increase $n \_t r i a l s$, or if you want to work with a different dataset you can specify its definition in ds_json.

The script will automatically check for changes to the dataset parameters and definitions of the noise classes (corruptions) and regenerate the datasets if they are outdated. The results will be written to the console and testresults/Test [current time]. Additional output may be written to testresults/log.txt.

\section{Generating Datasets}

Datasets are defined through both a dataset definition file and a noise classes specification. A dataset definition file (for example degradation/basic_dataset. j jon) contains an object with the following fields:

- "name", the name of the dataset. This is used as the name of the folder that contains the dataset and the filename of trained . noiseclasses files in classifier/models.

- " train", the number of files in the training set. If less than 1 it is used as a fraction.

- "test", the equivalent for the testing set.

- "weights", a mapping of noise class identifiers to fractions. Describes the ratio of files which are given the corruption.

- "pipeline", a list of noise class or operation (see below) identifiers. The list describes the order in which degradations are applied. All identifiers in the weights mapping must occur in the pipeline list.

Additionally, two additional fields may be present:

- "operations", a list of objects similar to noise classes. An operation has an identifier ("name") and a list of degradations. If an operation is present in pipeline it is applied to all samples at that point unless the sample has been assigned incompatible labels.

- "incompatible", a list of noise class and operation identifier combinations. These are resolved as follows: 
1. Labels are randomly assigned to samples according to weights.

2. The samples which are assigned all the noise class identifiers present in a combination have those labels replaced with only one of them. The probability of a label being chosen to remain is proportional to the label's weight.

3. If there is an operation identifier present in the combination, that operation is omitted for all samples in step 2.

A combination must have at least two identifiers, of which at most one may be an operation identifier.

The degradations in each noise class is not defined in the dataset definition but in a noise classes specification (normally noise_classes/noise_classes. json). In retrospect this may have been a poor design choice, but here we are. This is a file containing a list of noise class objects. The parts relevant for dataset generation are the "id" and "degradations" fields.

The degradations list contains one or more objects specifying the degradation function in the ADT that will be invoked, as well as its parameters. name must be one of the names listed in degradation/degradations . py, for example "applyHighpassfilter" or "addSound". The arguments accepted varies by degradation (for example, addSound takes normalizeoutputAudio and snrRatio) and are listed in the degradation's Matlab file. Additionally, the intermediate script degradation/create_samples.m interprets some arguments (such as addSoundFile, i.e. the soundfile being added to the sample). Arguments are generally floats or strings, but certain special objects can be used to get an element of randomness. These are:

- $\{$ "randomNoiseFile": "string"\}, will get a random file from noise/string belonging the corresponding set (training or testing).

- $\{$ "randomchoice" : [array]\}, will pick a random element from the array provided.

- $\{$ "randomRange": [array of length 2]\}, will pick a random float in the provided range.

- $\{$ "randomRangeInt": [array of length 2]\}, will pick a random integer in the provided range.

The degradations list is interpreted the same way whether it is part of a noise class or an operation.

If you are using the experiments . py script to run your tests, datasets are generated and updated for you. However, you can also use degradation/create_dataset. py directly to create your datasets yourself. Try degradation/create_dataset.py --helpordegradation/create_dataset.py create - help for information on specific usage.

\section{Adding Corruptions}

Assume that we want to add a corruption that applies a DC offset to the waveform. As there is no such degradation in the ADT we need to implement a custom degradation. We start by creating a copy of template . $m$ in degradation/custom-degradations. Let us name this copy degradationUnit_addDcoffset.m. Open the file in a text editor or Matlab. 
Replace template with degradationUnit_addDcoffset in the function name on the first row and in the comment below. Add the current date, your name and a description of what the degradation will do. Reasonable arguments to our degradation is the amount of bias and whether we take into account the existing bias or not. We add the two arguments bias, with a default of $\odot .05$, and relative, with a default of $\odot$.

To add the bias we replace $\mathrm{f}$ _audio_out = f_audio; in the main program section with the following:

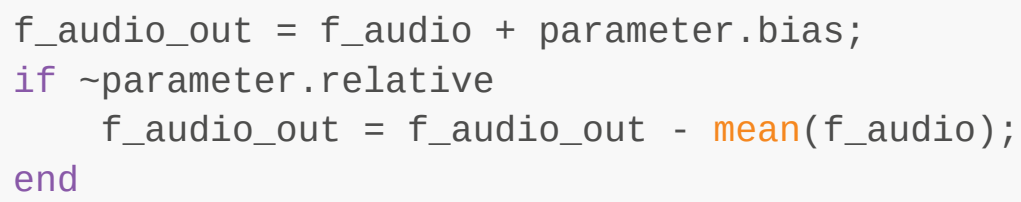

The entire file should now look like this:

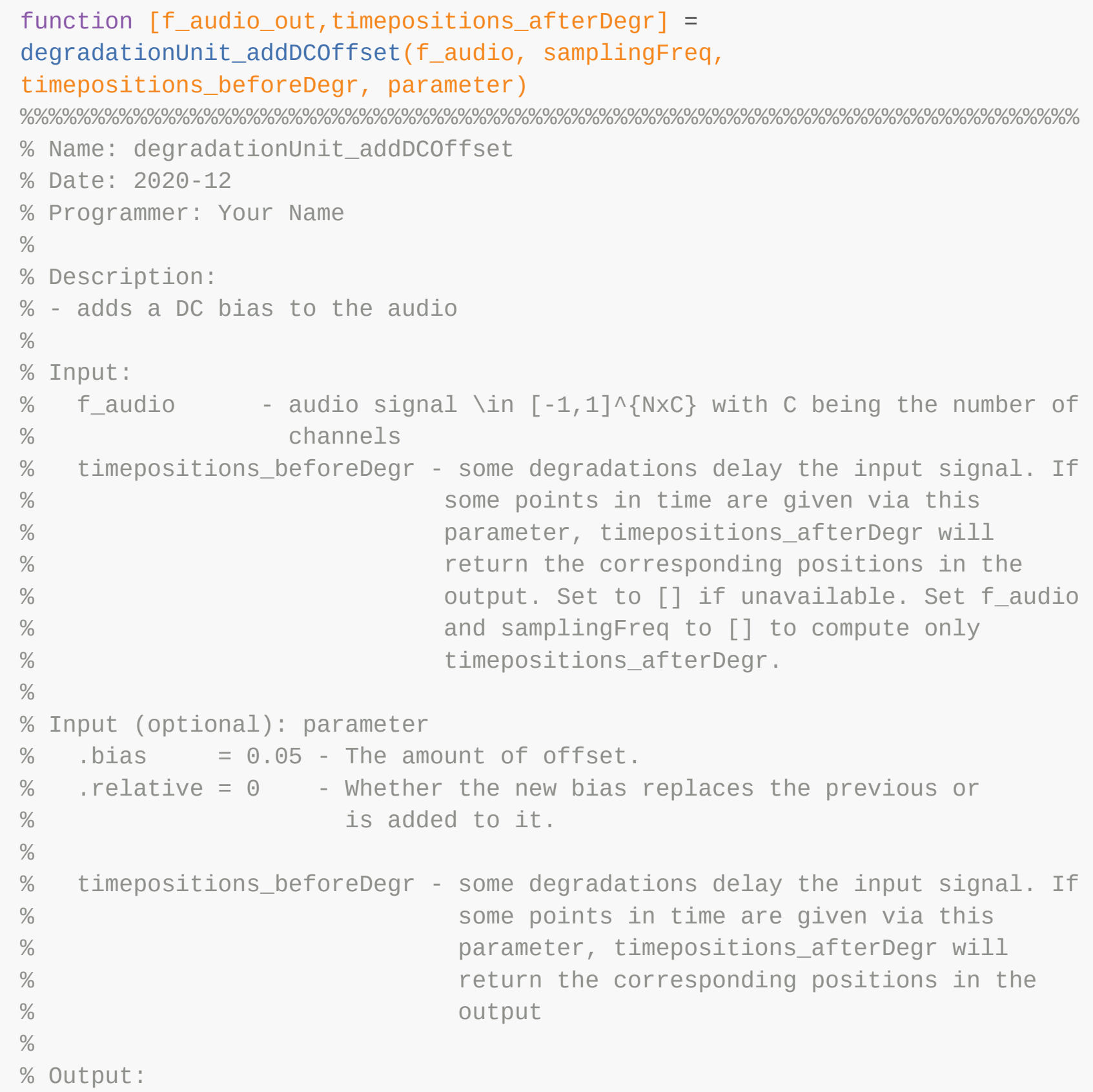




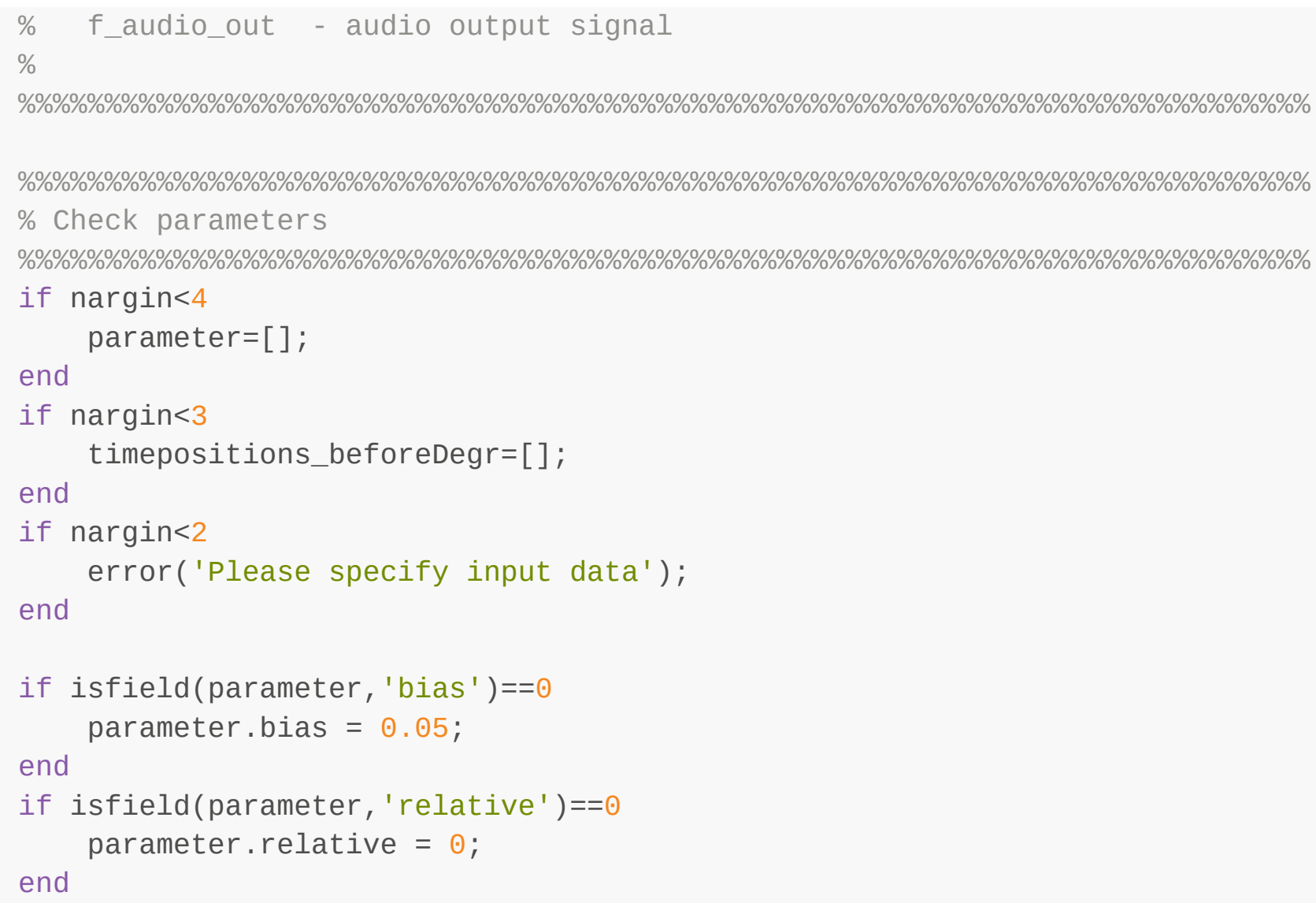

As we have named our file with the prefix degradationUnit_and put it in the custom-degradations folder, create_dataset . m will automatically find our Matlab function for us. However, we also need to make sure the Python script knows our degradation exists. To do this we open degradation/degradations . py and add "addDCoffset" to the DEGRADATIONS constant. It should now read:

DEGRADATIONS $=($

"", "pad", "addSound", "applyImpulseResponse", "adaptiveEqualizer", "applyMfccMeanAdaption", "normalize", "applyMute", "applySoftclipping", "addNoise", "applyAliasing", "applyclipping", 
"applyclippingAlternative", "applyDelay",

"applyDynamicRangeCompression", "applyHarmonicDistortion",

"applyHighpassFilter", "applyLowpassFilter", "applyspeedup",

"applyWowResampling", "addInfrasound", "addDcoffset" )

Next, we have to add our new corruption to noise_classes/noise_classes . j son. To introduce some variety we set the bias argument to a random value between $\odot .02$ and $\odot .07$. As the offset is likely more detectable in the time domain than the frequency domain we use a histogram feaure and a GMM classifier for now. Add the following to the end of the list, right before the final ] symbol:

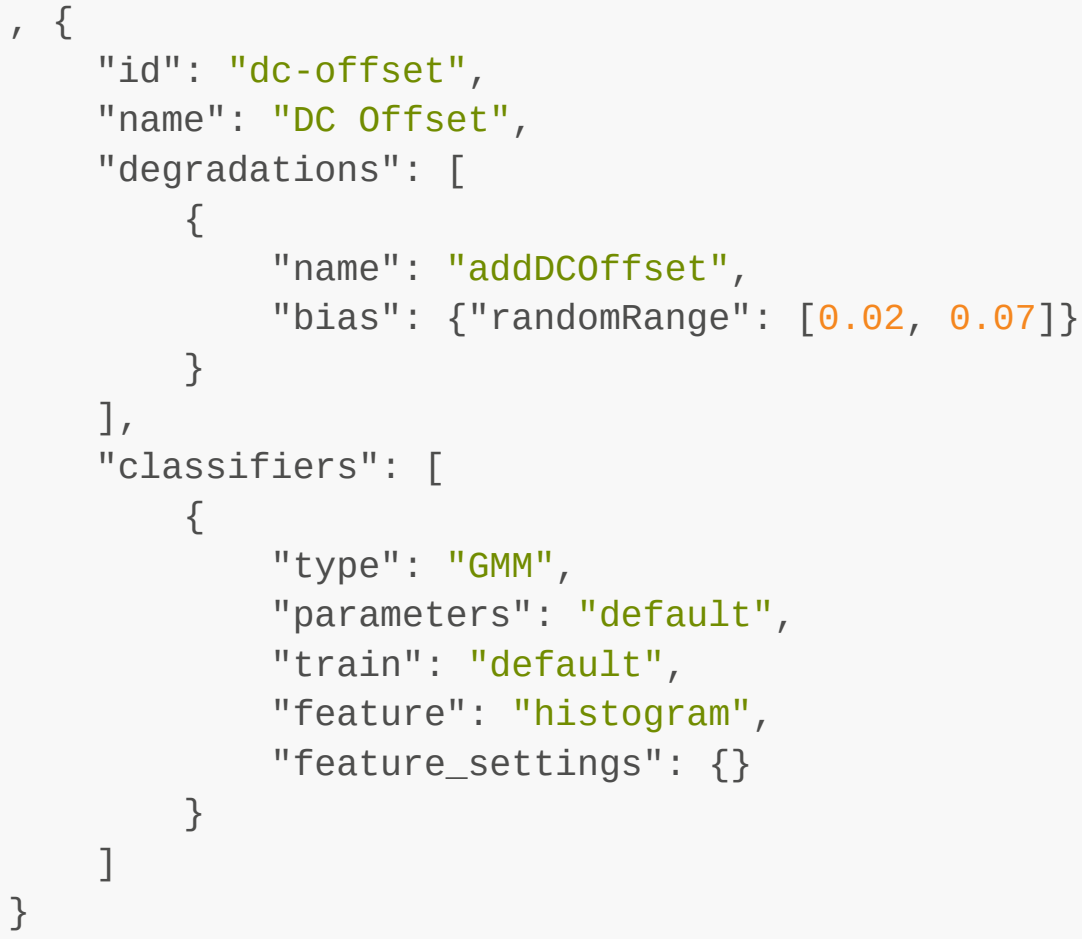

Finally, we also have to update our dataset specification with the new noise class - otherwise no samples will have the corruption, and no classifiers can be trained to detect it. Open degradation/basic_dataset.json and add "dc-offset": 0.125 to "weights". Also add "dcoffset" to "pipeline" before "clipping-soft". The result should look like:

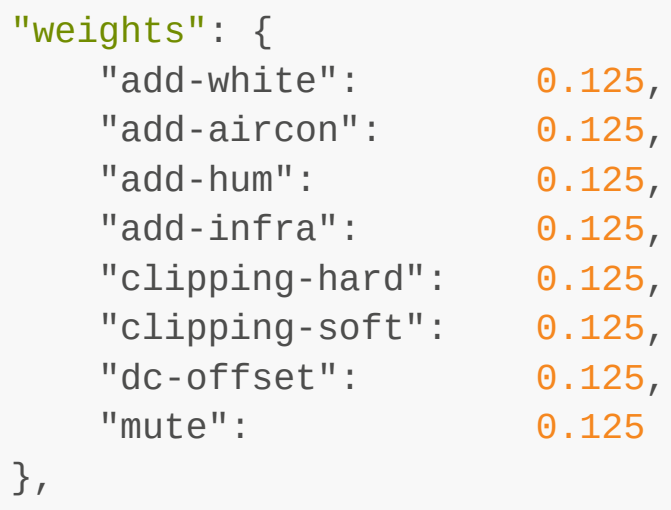




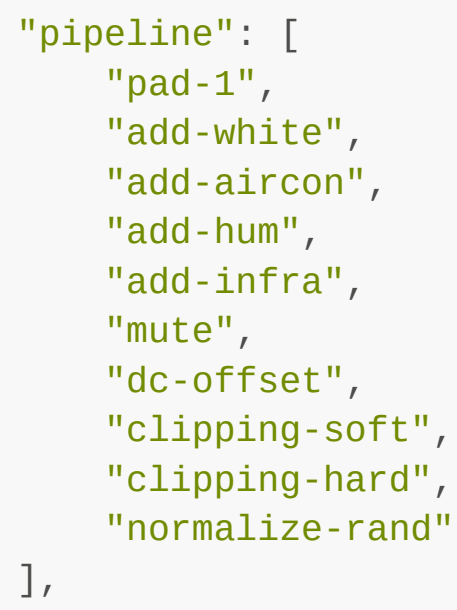

Now you are all set to try out the new corruption! Run experiments . py to generate the dataset and train/test the classifiers. For reference I get a $96.9 \%$ balanced accuracy. (Note, however, that we have only allowed a positive bias.)

To summarize, the steps are as follows:

1. If needed, create a Matlab script to perform some operation on the audio signal. You can use degradation/custom-degradations/template . m as a starting point.

2. Register the script in degradation/degradations . py.

3. Create a new noise class in noise_classes/noise_classes . json.

4. Add the noise class identifier to a dataset's weights and pipeline.

\section{Adding Features}

In the previous section we used a histogram feature to detect the DC offset corruption. While this certainly may be a good starting point, a more straightforward approach would be to use the mean of the signal as a feature. We thus need to implement it and add it to the appropriate files.

Open features/custom . py. Every feature function receives the list of files from which features will be extracted as the first argument. Settings for the computation can be provided via keyword arguments, which will be filled in by the fields in the noise class' "feature_settings" object. For instance, we can specify the number of bins in the histogram feature by writing e.g.

"feature_settings": \{"n_bins": 10\}

In our current case, with the mean feature, there is not really any more information we need to compute the feature, so we accept no keyword arguments. At the bottom of custom . py we add the following method definition: 


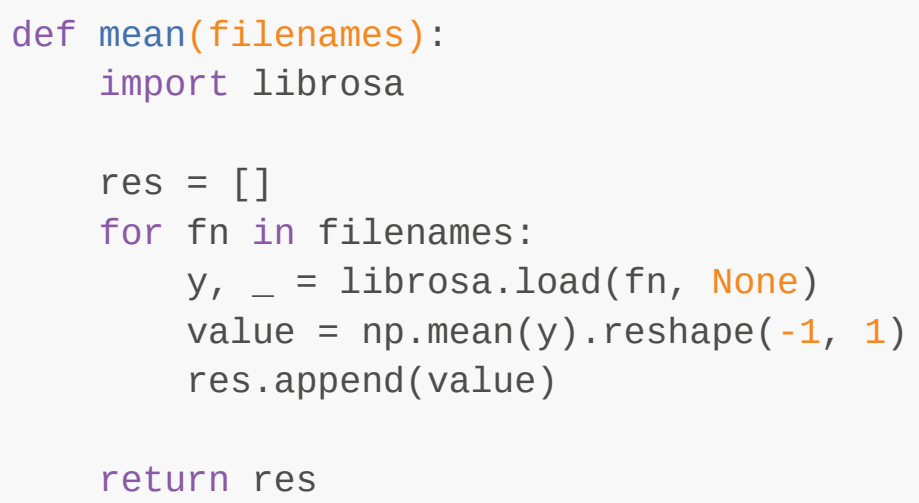

We use the librosa module to read each sample, and numpy to compute the mean. The return value of a feature function must be a list of numpy arrays, one for each file. The arrays should have the shape ( $T$, dims ), where $T$ is the length of the time sequence and dims number of feature dimensions. dims should be the same for each array, but $T$ may vary. In our case we are only computing a scalar, so we convert it into a $1 \times 1$ array. (The arrays also need to be of the float 32 type. While this is the default in many cases, certain librosa spectrum computations return float64.)

The next step is to let features/feature_extraction. py know of our new method. At the start we add mean to the import statement from . custom:

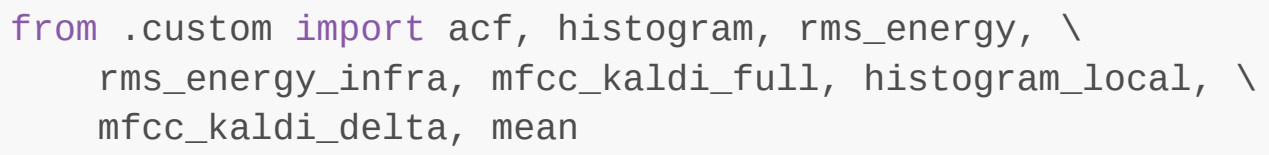

We also add it to the FEATURES constant:

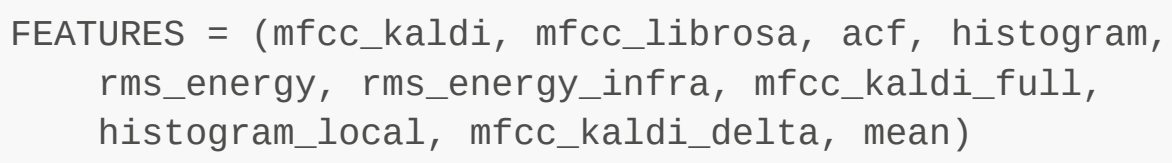

While we are editing this file it is worth to note the CACHE_SIZE constant defined on the row below FEATURES. Extracting features can take a long time, and if we are training multiple classifiers on the same datasets and using the same features it would be redundant to keep recomputing them. Instead we save a number of computed features to the cache folder (at most CACHE_SIZE, after which the oldest ones are removed). While this is practical during experiments it is less so if you are debugging a feature method, in which case it is suggested to set CACHE_SIZE to $\odot$ or to clear it manually by deleting the files after each run.

Now, the only thing to do is to swap out the histogram and replace it with the mean. In noise_classes/noise_classes.json, replace "feature": "histogram" with "feature": "mean" in the DC offset noise class. If you re-run the experiment you should see that experiment . py does not need to regenerate the dataset, and that the GMM classifier for dc - off set now uses mean 
features. When testing this the balanced accuracy increases to $99.97 \%$ for me, but as there are elements of randomness the results may vary slightly.

\section{Voice Activity Detection}

For corruptions that only arise during speech it may be interesting to filter out silent segments, and for those that are related to background noise it may be beneficial to remove segments where the speech is dominant. To this end there is a way to split the recordings using a Voice Activity Detector (VAD). By adding a "vad" field to an object in the "classifiers" array of a noise class you indicate that a VAD will be used, and can specify settings which are passed to features/vad . py.

Both frame_length and aggressiveness are required arguments to the VAD. Available settings are:

- frame_length, the length of the VAD analysis frame in milliseconds. It must be one of 10,20 , or 30 .

- aggressiveness, how likely the VAD is to consider a frame non-speech. Must be one of $0,1,2$, or 3 .

- inverse, whether to return the voiced (false) or the unvoiced (true) segments. Defaults to false.

- smooth_n, the length of the smoothing window used. If not set, smoothing is not used at all. Smoothing computes a running average of the VAD verdict of the smooth_n nearest frames. This ensures that a single mislabeled frame does not affect the segmentation.

- smooth_threshold, the threshold that must be reached by the running average to toggle between the voiced/unvoiced state. For example, if smooth_threshold $=0.8$ then $80 \%$ of the frames must be labeled voiced for a voiced segment to start, after which $80 \%$ of frames must be labeled unvoiced to end the segment. Defaults to $\odot .5$.

- min_length, the minimum length of a segment in milliseconds. Segments that are shorter than this are discarded. Defaults to $\odot$, which will include all segments.

\section{Adding Classifiers}

Assume that we want to see how a new classification algorithm fares against the others in the test. We choose the Decision Tree (DT) algorithm which conveniently is implemented in scikit-learn, a module we are already using. To interface with the feature extraction and other parts of the tool all algorithms must inherit from classifier/model . py. We start by creating a new file, model_dt . py in the classifierfolder with the following content:

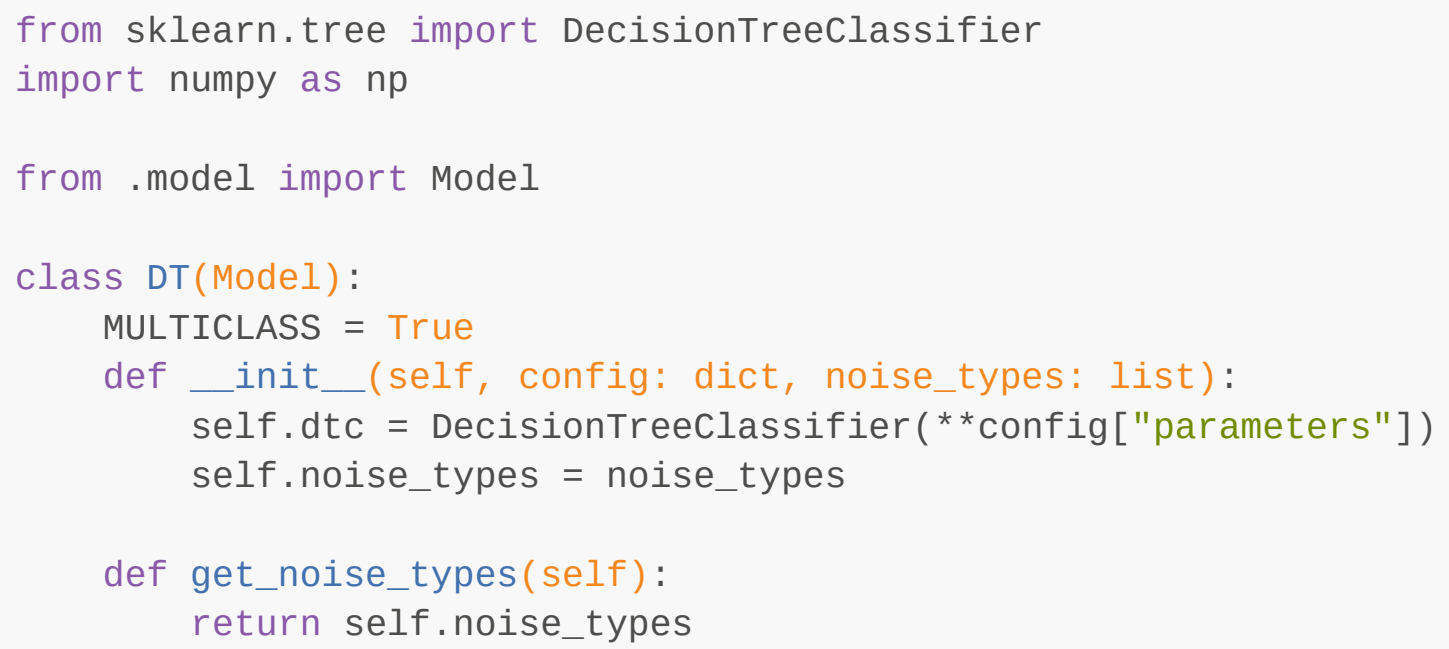




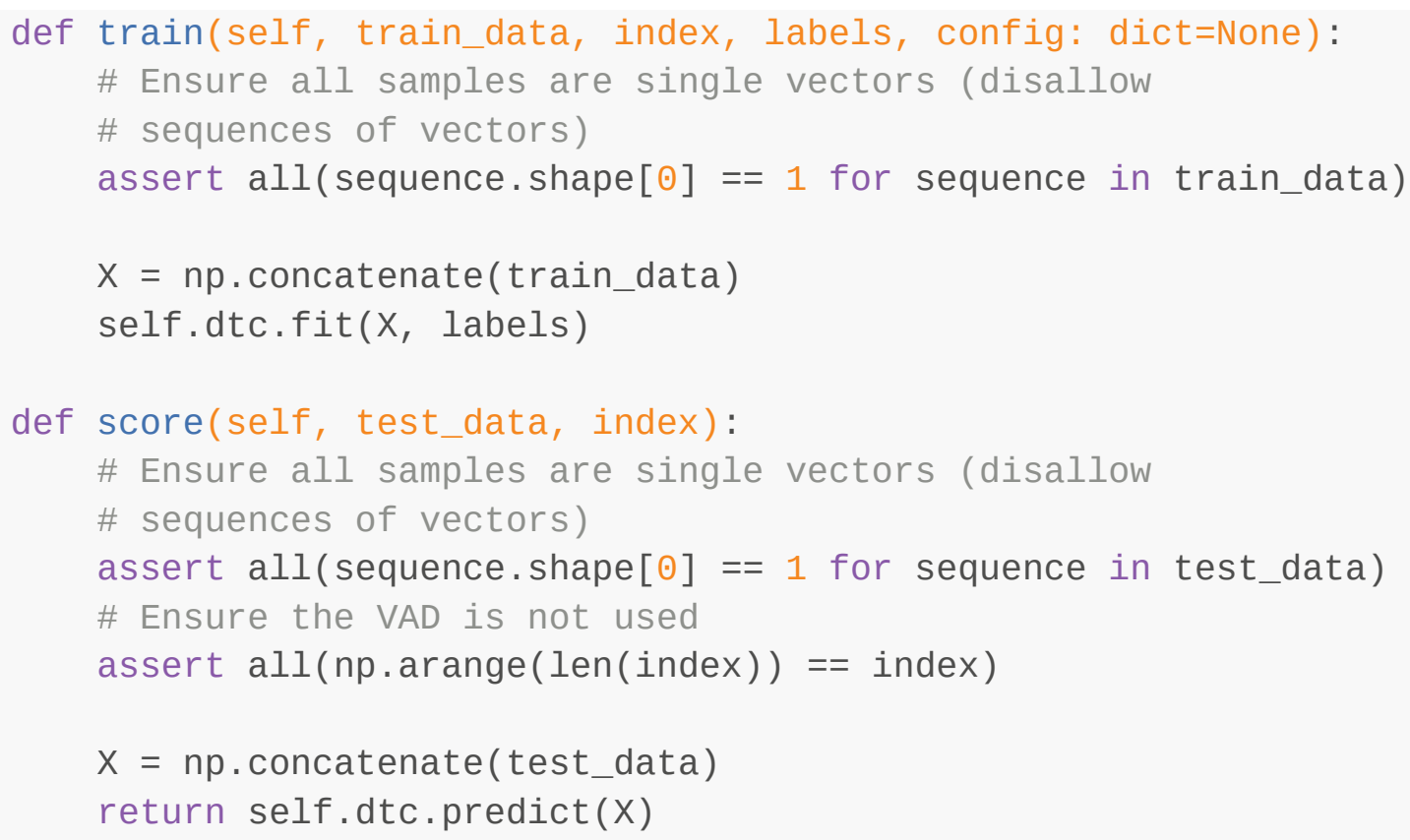

For brevity we take some shortcuts in this classifier by disallowing sequences of vectors (such as MFCC features) and VAD splitting.

To make the classifier usable we need to add it to noise_classes/interface . py, where the names are resolved. In the beginning of the file there are a number of rows where the other classifiers are imported. Add a line importing the DT classifier and add it to available_models like so:

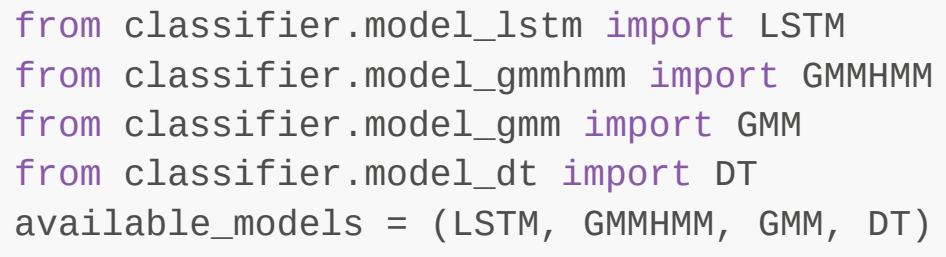

Before we can use it, however, we also need to add an entry specifying the (default) arguments to the classifier.

According to the scikit-learn documentation the DecisionTreeClassifier takes a number of possible keyword arguments, such as criterion or max_depth. We can provide our own defaults to these by adding them to classifier/defaults. json, which is done by adding the following before the last curly brace: 


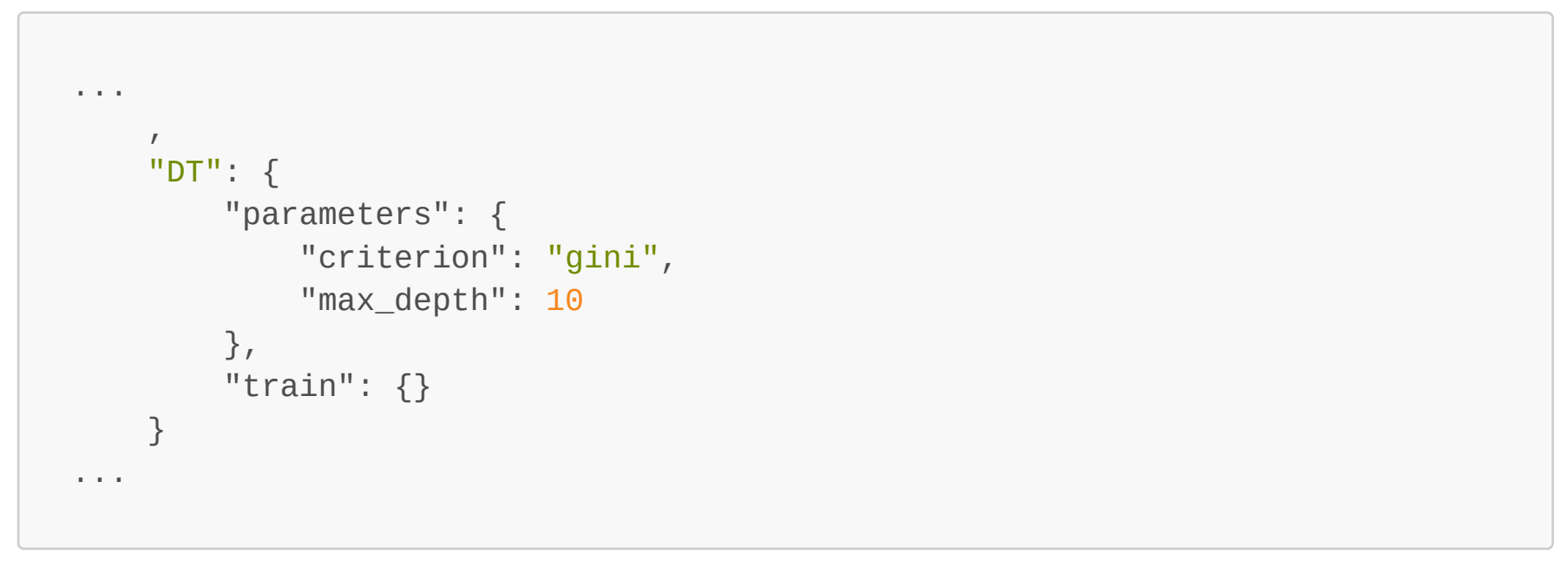

Next, open noise_classes/noise_classes . j son and change the classifier type of DC offset from GMM to DT. Also change the feature type back to histogram if you modified it in the last step. If you did not implement the DC offset corruption you can use one of the clipping corruptions instead.

A decision tree will now be used to detect the corruption. Note that the classifier specification also contains "parameters" and "train", just like defaults. j son. Setting these to "default" will copy the settings from defaults. j son, but if we want to override a setting specifically for a certain corruption it can be done here. In that case we need to specify all the fields in the category we are replacing, for example setting

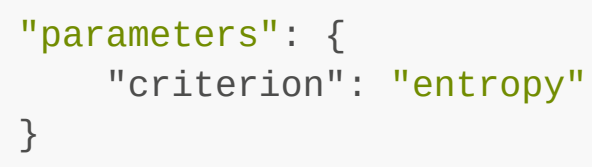

in noise_classes. json would change the criterion argument to "entropy", but also unset the max_depth argument.

Now, having implemented DT, added it to defaults. j son and interface. py, and specified it as the classifier for the DC offset, we are all set to test it out. Try running experiment s . py and see if the DT classifier performs as well as the GMM. For reference, I get a $99.7 \%$ balanced accuracy on DC offset.

The arguments train_data and test_data in the test ( ) and score ( ) methods of model_dt.py are the vector sequences returned by the feature extraction methods. Recall that they consist of numpy arrays of shape ( $T$, dims ), where dims is the same for all arrays. If the VAD is used there are in general multiple arrays per sample, corresponding to the different voiced or unvoiced segments in the recording. The index variable can be used to keep track of these: array test_data [i] belongs to file number index [i]. During labeling most existing classifiers score segments separately and then compute the weighted mean of the scores, which is used as the score for that recording. (Note that score ( ) should return one score per recording, not per segment, so test_data is generally longer than the returned score array when VAD filtering is used.) 


\section{Ensemble Classification}

The keen reader will have noticed that the "classifiers" field in a noise class is actually an array of objects. This is because you can specify multiple classifiers for the corruption which will vote on the final label. Simply add another classifier specification to the list - these do not have to use the same algorithm or even features. You can specify the weights of individual classifiers by adding a "weight" field (the default is 1), and indicate that the classifier should train on a bootstrapped sample by adding "bootstrap" : true. If you have multiple classifiers of the same type and want to average the score rather than the label during voting, you can add a field to the noise class specifying "classification_settings":

\{"average": "score"\}.

Note that since training is done separately for each classifier, the training time will increase considerably when ensemble classification is used. 

TRITA-EECS-EX-2021:50

www.kth.se 